\title{
Applications of Attenuation Tomography to Imperial Valley and Coso-Indian Wells Region, Southern California
}

\author{
Phyllis Ho-LIU, HiRoo Kanamori, AND RoBert W. Clayton \\ Seismological Laboratory, California Institute of Technology, Pasadena
}

\begin{abstract}
Spatial variations in seismic wave attenuation were tomographically imaged from observed $S$ to $P$ wave amplitude ratios in the Imperial Valley and Coso-Indian Wells regions of southern California. In the Coso-Indian Wells region a highly attenuating body ( $S$ wave quality factor $Q_{\beta} \approx 30$ ) coincides with a slow $P$ wave anomaly mapped by Walck and Clayton (1987). This coincidence suggests the presence of a 3- to 5-km depth magmatic or hydrothermal body in the Indian Wells region. In the Imperial Valley, slow $P$ wave travel time anomalies and highly attenuating $S$ wave anomalies were found in the Brawley seismic zone at a depth of 8-12 km. The effective $S$ wave quality factor is very low $\left(Q_{\beta} \approx 20\right)$, and the $P$ wave velocity is $10 \%$ slower than the surrounding areas. These results suggest either magmatic or hydrothermal intrusions, or fractures at depth, possibly related to active shear in the Brawley seismic zone. This attenuation tomographic technique is shown to be useful in delineating the spatial variations in seismic wave attenuation and in estimating the effective seismic quality factor of attenuation anomalies.
\end{abstract}

\section{INTRODUCTION}

Back projection tomography is a technique that utilizes an iterative approach to a solution of an inverse problem. It has recently been applied to travel time data of seismic waves to invert them for velocity perturbations [Humphreys et al., 1984; Hearn and Clayton, 1986a b; Walck and Clayton, 1987; R. W. Clayton and R. P. Comer, unpublished manuscript, 1987; R. P. Comer and R. W. Clayton, unpublished manuscript, 1987]. In this paper a similar method is applied to amplitude data to locate attenuating bodies in the crust. An attenuating body decreases the amplitude of seismic waves passing through it, just as a low-velocity body lengthens the travel time. Previous work on estimating the quality factor of a medium includes the reduced spectral ratio technique [Ward and Young, 1980] which inverts the spectral data directly for the $P$ wave attenuation, spectral ratio analysis which calculates the quality factor for $S$ waves [Kobayashi et al., 1986; Sudo, 1987], and cross-hole seismic imaging at two sites which reconstructs the perturbations of $\boldsymbol{P}$ velocities between the holes [Wong et al., 1983]. This paper describes the technique of the tomographic inversion of amplitude data and presents two applications. The results from travel time tomography and attenuation tomography are also compared.

\section{TraVel TME TOMOgRaPHY}

Standard travel time tomography uses the relation between slowness and travel time and is applied to travel time perturbations (R. P. Comer and R. W. Clayton, unpublished manuscript, 1987). The seismic travel time is given by the relation

$$
\int s d l=t
$$

where $t$ is the travel time from source to receiver, $d l$ is the incremental ray length, and $s$ is the slowness along the ray

Copyright 1988 by the American Geophysical Union.

Paper number 7B5033.

0148-0227/88/007B-5033\$05.00 path. However, since the ray path depends on 8 , this equation is nonlinear with respect to $s$. A linear system can be obtained by the use of perturbations in $t$ and $s$ [Fawcett and Clayton, 1984]. Equation (1) then becomes to first order

$$
\int \Delta s d l=\Delta t
$$

where $\Delta s$ and $\Delta t$ are the perturbations in slowness and travel time, respectively. If the medium is divided into blocks, equation (2) can be written as

$$
\sum \Delta s, l_{s,}=\Delta t_{\mathrm{i}}
$$

where $j$ is block number, $i$ is ray number, and $l_{v}$, is the length of the $i$ th ray in the $j$ th block.

\section{ATTENUATION TOMOGRAPHY}

The tomographic method described by R. P. Comer and R. W. Clayton, (unpublished manuscript, 1987) can also be applied to amplitude data to determine attenuation structure of the medium. We divide the medium into $N$ blocks and let $Q$, be the quality factor in the $i$ th block. Then the amplitude of a ray $j, A$, is given by

$$
A_{s}=A_{0}, \prod_{i=1}^{N} \exp \left(-\frac{\pi f t_{t s}}{Q_{t}}\right)
$$

where $A_{0 j}$ is the initial amplitude of ray $j, f$ is the frequency, $t_{z}$ is the travel time of ray $j$ through the $i$ th block, and $N$ is the total number of blocks in the medium. We set $t_{i j}$ (and $l_{i j}$ below) to be zero for blocks not crossed by the $j$ th ray.

Rearranging (4) and substituting $t_{i j}$ by $l_{1} s_{1}$, with $s_{1}$ denoting the slowness of the $i$ th block, we have

$$
\frac{A_{J}}{A_{0_{j}}}=\prod_{i=1}^{N} \exp \left(-\frac{\pi f l_{i j} s_{i}}{Q_{i}}\right)
$$

or

$$
\ln \left[\frac{A_{j}}{A_{0 j}}\right]=\sum_{v=1}^{N}\left[\frac{-\pi f s_{1} l_{v y}}{Q_{v}}\right]=-\sum_{s=1}^{N} m_{v j}\left[\frac{f}{Q_{v}}\right]
$$


where $m_{i g}$ is $\left(\pi s_{i} l_{1,}\right)$. Denoting $\left[-\ln \left(A, / A_{0},\right)\right]$ and $f / Q$, by $a$, and $g$, respectively, we have, from (6),

$$
a,=\sum_{i=1}^{N} m_{i}, q_{1}
$$

Equation (7a) has the same form as equation (3) with $q_{t}$, $a_{j}$ and $m_{i j}$ replacing $\Delta s_{i}, \Delta t_{j}$, and $l_{i j}$, respectively. We can then use the algorithm developed by $R$. P. Comer and R. W. Clayton, (unpublished manuscript, 1987) to solve for the unknown $q$, in the medium:

$$
\begin{aligned}
& q_{1}{ }^{k+1}=q_{i}{ }^{k}+\frac{\sum_{j}\left(\frac{a_{j}^{k}}{L_{j}}\right) m_{\jmath}}{\mu+\sum_{j} m_{\jmath 1}} \\
& a_{,}{ }^{k}=a_{1}{ }^{0}-\sum_{1} m_{j,} q_{1}
\end{aligned}
$$

where $k$ denotes the index of iteration, $L$, is the total length of ray $j$, and $\mu$ is a damping factor to reduce the effects of less constrained model parameters and to stabilize the solution (R. P. Comer and R. W. Clayton, unpublished manuscript, 1987; P. Ho-Liu et al., unpublished manuscript, 1987).

The effective quality factor in the medium can be calculated from (7). However, the block slowness $s_{1}$ is not known a priori. We can approximate $s$, by the slowness of the $i$ th block in the reference velocity model used to trace rays through the medium. Also, $a$, is not known because the initial amplitude of the ray is unknown. We obtain $a_{\text {, }}$ by the following analysis. Here we denote the observed $S$, $P$ wave amplitude by $A_{s, p}$, the initial $S, P$ wave amplitude by $A_{s, p}^{0}$, the amplitude radiation pattern of $S$ and $P$ waves by $R_{0, p}$, the instrument response for $S$ and $P$ waves by $I_{s, p}$, the geometrical spreading factor for $S$ and $P$ waves by $G_{s, p}$, the frequency by $f$, the quality factor for $P$ and $S$ waves by $Q_{\alpha_{1} \beta}$, the distance traversed by seismic wave by $l$, and the slowness parameter by $s_{\alpha_{0} \beta}$. The observed $P$ and $S$ amplitudes are given by

$$
\begin{aligned}
& A_{p}=A_{p}{ }^{0} R_{p} I_{p} G_{p} \exp \left(\begin{array}{lll}
-l s_{\alpha} f & \frac{\pi}{Q_{\alpha}}
\end{array}\right) \\
& A_{a}=A_{a}{ }^{0} R_{a} I_{a} G_{\alpha} \exp \left(-l s_{\beta} f \frac{\pi}{Q_{\beta}}\right)
\end{aligned}
$$

Dividing (9) by (8), we have

$$
\frac{A_{0}}{A_{p}}=\frac{A_{0}^{0}}{A_{p}^{0}} \frac{R_{0}}{R_{p}} \frac{I_{0}}{I_{p}} \frac{G_{0}}{G_{p}} \exp \left[-\pi f l\left(\frac{\theta_{\beta}}{Q_{\beta}}-\frac{s_{\alpha}}{Q_{\alpha}}\right)\right](10)
$$

If we assume that $I_{0} \approx k_{1} I_{p}$ (where $k_{1}$ is a constant), $G_{0} \approx G_{2}$, and $A_{0}{ }^{0} \approx k_{2} A_{2}{ }^{0}$ (where $k_{2}$ is $(\alpha / \beta)^{8}$ ) and let the quality factor for $P$ waves, $Q_{\alpha}$, be represented by $\eta Q_{\beta}(\eta$ is a constant), we then obtain

$$
\frac{A_{s}}{A_{p}}=k_{1} k_{2} \frac{R_{s}}{R_{p}} \exp \left\{-\pi f l\left[\frac{s_{\beta}}{Q_{\beta}}\left[1-\frac{s_{\alpha}}{\eta_{\beta} \beta}\right)\right]\right\}
$$

For normal crustal rocks, $\eta \approx 2.25$ and the ratio $8_{\alpha} / \eta s_{\beta}$ is approximately 1/4 [Anderson and Archambeau, 1964; Anderson, 1967]. If the attenuation is caused by the existence of fluid phase, the ratio is even smaller than $1 / 4$. We therefore neglect this term in the inversion. Some observational work indicates that $\eta$ in the midcrust can be as low as 1 [Rautian et al., 1978; Frankel, 1982]. If $\eta \approx 1$, we see from equation (11) that we only need to multiply the inverted quality factor by a factor of approximately 0.4 .

The constant $k_{1}$ is determined by the response of the seismograph (in this case, vertical component instruments are used) to the incident $P$ waves and $S V$ waves. It depends on the incidence angle of the wave at the station. Because the velocity of the medium near the surface is very low, the incidence is near vertical so that the vertical component seismograph is not as sensitive to $S V$ waves as it is to $P$ waves. We therefore expect $k_{1}$ to be a small number, probably in the range of 0.1-0.5. We cannot calculate the exact value of $k_{1}$ because we do not know the actual $P$ and $S$ velocity profiles in the areas. Errors in $k_{1}$ propagate throughout the inversion. We include this ratio as an unknown parameter in the inversion and vary it, thereby obtaining the sensitivity of the inversion results to $k_{1}$.

The constant $k_{2}$ is determined by the $P$ and $S V$ velocities at the source and is approximately 5 for most rocks. Therefore we expect the product $k_{1} k_{2}$ to be on the order of 1. Since the value of $k_{1}$ is uncertain, we perform the inversion for several trial values of $k_{1} k_{2}$ and compare the results. With these assumptions, we obtain

$$
\frac{A_{s}}{A_{p}} \approx k_{1} k_{2} \frac{R_{s}}{R_{p}} \exp \left(-\pi f l \frac{s_{\beta}}{Q_{\beta}}\right)
$$

or

$$
-\ln \left(\frac{A_{0} / A_{\beta}}{k_{1} k_{2} R_{0} / R_{p}}\right)=\pi s_{\beta} l \frac{f}{Q_{\beta}}
$$

Equation (12) can be cast into discrete form as equation (7) with $a_{1}$ replaced by $-\ln \left[\left(A_{0} / A_{p}\right) /\left(k_{1} k_{2} R_{0} / R_{p}\right)\right]$, $q_{1}$ by $f / Q_{1}$, and $m_{1}$ by $l_{1,} \pi s_{\beta_{2}}$. The tomographic technique can be readily applied to solve (12) for the attenua-

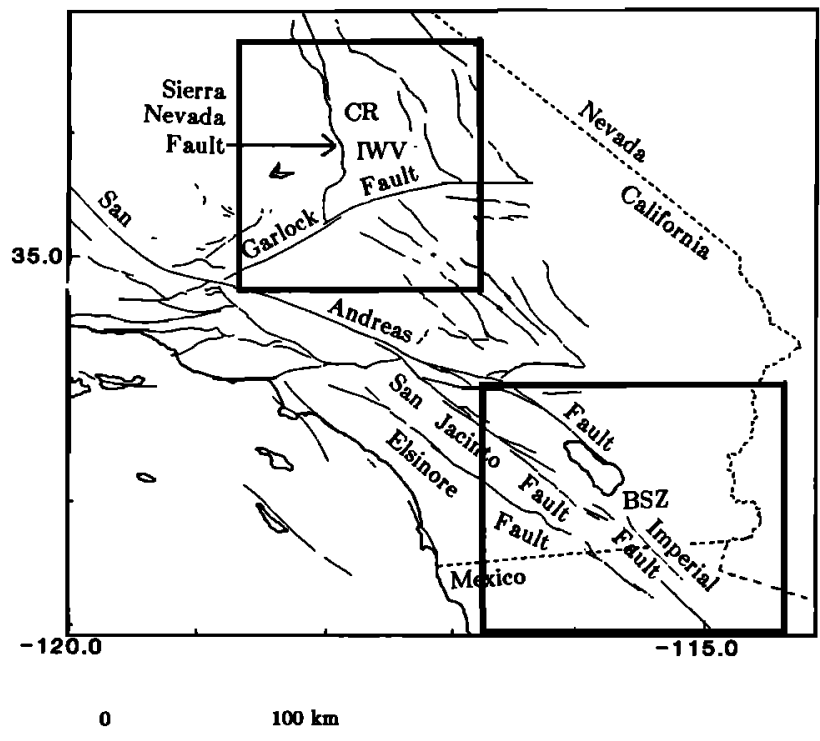

Fig. 1. Boxes enclose the attenuation tomography study areas in Coso-Indian Wells Valley and Imperial Valley, southern California. Major faults are indicated and the abbreviations are CR,Coso Range; IWV,Indian Wells Valley and BSZ,Brawley seismic zone. 


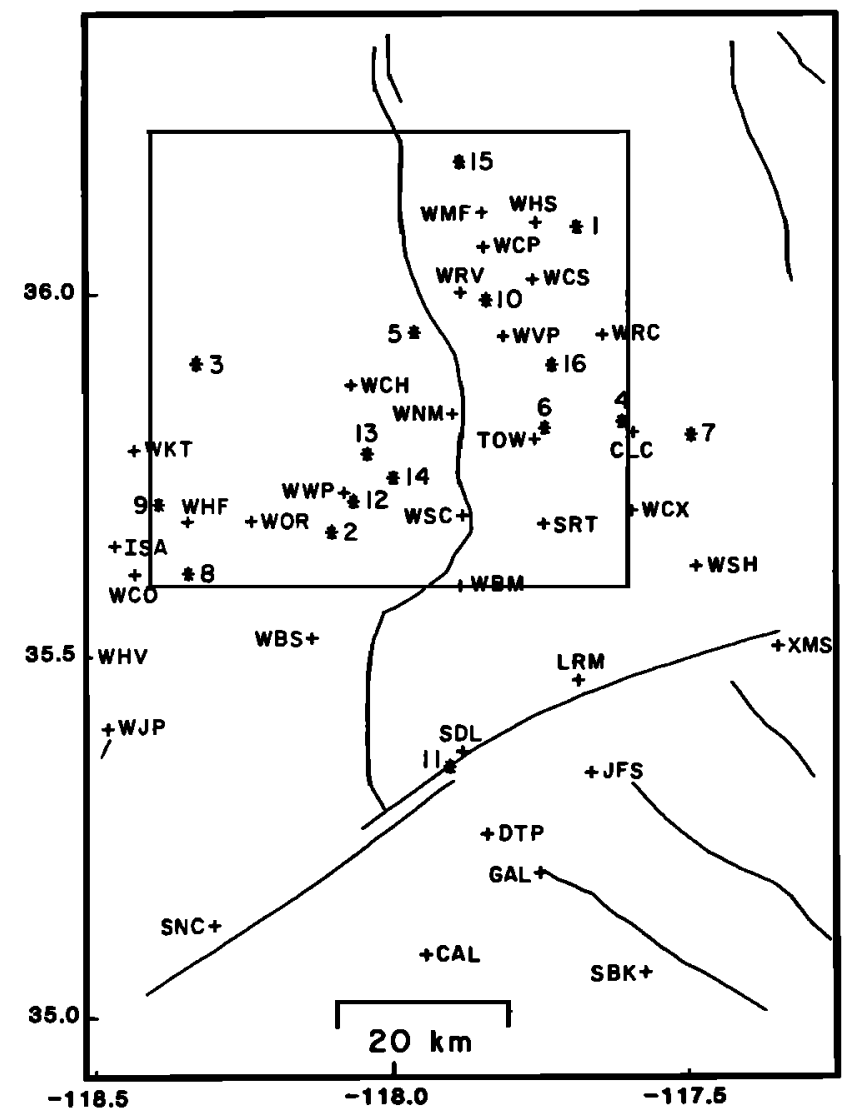

Fig. 2. Numbers indicate the locations of the 16 earthquakes used in the attenuation inversion. Station codes show the locations of the Southern California Array stations used in the Coso region study. Inner box is the study area of travel time inversion by Walck and Clayton [1987].

tion parameter $f / Q$, for the appropriate frequency range of the data.

\section{APPLICATIONS}

We have applied the method to data sets obtained for the Coso-Indian Wells Valley and the Imperial Valley, southern California (Figure 1). These two areas have several features in common. Both are sediment-filled basins in active tectonic areas that are local spreading centers or regions of extensional stresses [Hill, 1977; Weaver and Hill, 1978]. Earthquake swarms are common in both areas. Magmatic activity has been suspected to cause such swarm activity. However, the three-dimensional structure in these two areas is not known in sufficient detail to determine whether the swarms are due to magmatic, hydrothermal, or other causes. The attenuation tomographic method described above helps us to detail the three-dimensional $Q$ structure of the underlying medium, thereby adding a constraint on the characteristics of the causes of earthquake swarms.

For Coso-Indian Wells Valley we used the 16 earthquakes shown in Figure 2, which were also used by Sanders et al. [1988] for $S$ wave screening studies (Figure 4). Many other earthquakes in the same area could have been used in the study. We limit our choice of events to these 16 earthquakes because they provide an even depth and azimuthal coverage. The amount of computation can be kept to a reasonable level. Depths of these 16 earthquakes are listed in Table $1 a$. These earthquakes are relocated individually [Sanders et al., 1988]. In the attenuation inversion we used the same block size $(2 \mathrm{~km} \times 2 \mathrm{~km} \times 0.2 \mathrm{~km})$ as that used in the travel time inversion of Walck and Clayton [1987]. There were 72 blocks in the E-W direction and 72 blocks in the N-S direction, covering an area of $144 \mathrm{~km} \mathrm{x} 144 \mathrm{~km}$. The depth of the model was spanned by 75 blocks, so the model extended down to $15 \mathrm{~km}$. Since the ray-tracing code that we used requires a nonzero velocity gradient in the model, we used the small $(0.2 \mathrm{~km})$ block dimension in the depth direction to accommodate the velocity discontinuities in the model shown in Figure 7a. Since we do not expect a spatial resolution of $0.2 \mathrm{~km}$ from our data set, we averaged the results over several layers and present the final results in 1 or $2 \mathrm{~km}$ thick depth slices.

A data set of 15 earthquakes was chosen from an initial set of more than 200 earthquakes for both the $P$ wave travel time and $S$ wave attenuation inversions in the Imperial Valley (Figure 3). The earthquake depths are listed in Table $1 b$. All earthquakes used in this study are relocated individually using the layered-velocity model shown in Figure 6b. Similar to the case of Coso-Indian Wells region, other earthquakes could have been used in the inversion, but we limit our choice to a relatively small number of events with a good coverage of azimuth and depth to keep the amount of computation at a reasonable level. There are events outside the Brawley area that could have been selected. However, most of these events were not recorded on the stations within the area of interests. The station distribution is not as dense in this area as in Coso, forcing us to use more events in the Brawley area to maintain enough resolution in the Brawley seismic zone.

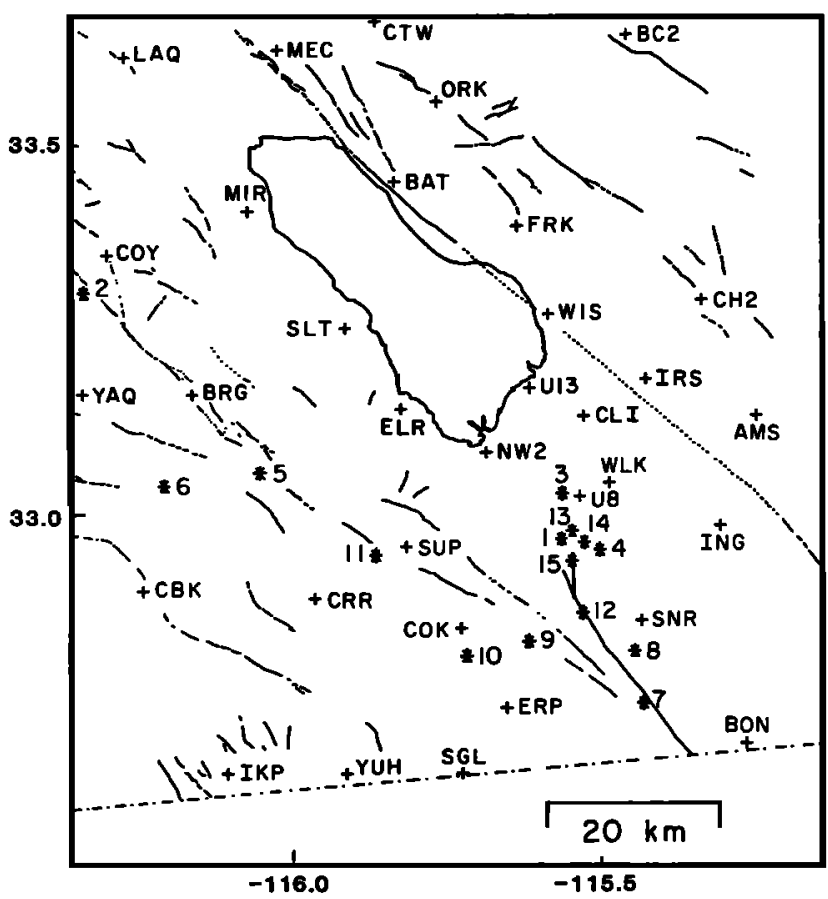

Fig. 3. Numbers indicate the locations of the 15 earthquakes used in the attenuation inversion. Station codes show the locations of the Southern California Array stations used in the Imperial Valley study. 
TABLE 1a. List of Dates and Hypocenters of 16 Earthquakes Used in the Coso-Indian Wells Region Study

\begin{tabular}{|c|c|c|c|c|}
\hline Event & Date & Latitude & Longitude & Depth,km \\
\hline 1 & Oct. 30,1983 & $36^{\circ} 06.88^{\prime}$ & $-117^{\circ} 40.35^{\prime}$ & 3.2 \\
\hline 2 & Feb. 24, 1984 & $35^{\circ} 41.11^{\prime}$ & $-118^{\circ} 06.02^{\prime}$ & 10.5 \\
\hline 3 & Feb. 24, 1984 & $35^{\circ} 55.10^{\prime}$ & $-118^{\circ} 19.27^{\prime}$ & 5.5 \\
\hline 4 & Jan. 9, 1984 & $35^{\circ} 49.87^{\prime}$ & $-117^{\circ} 36.29^{\prime}$ & 7.5 \\
\hline 5 & Jan. 27, 1984 & $35^{\circ} 57.44^{\prime}$ & $-117^{\circ} 57.93^{\prime}$ & 9.8 \\
\hline 6 & $\operatorname{Jan} .20,1984$ & $35^{\circ} 49.34^{\prime}$ & $-117^{\circ} 44.64^{\prime}$ & 6.8 \\
\hline 7 & Oct. 6, 1983 & $35^{\circ} 48.72^{\prime}$ & $-117^{\circ} 29.72^{\prime}$ & 6.5 \\
\hline 8 & Feb. 2, 1984 & $35^{\circ} 37.75^{\prime}$ & $-118^{\circ} 20.48^{\prime}$ & 13.6 \\
\hline 9 & July 24, 1983 & $35^{\circ} 43.48^{\prime}$ & $-118^{\circ} 24.46^{\prime}$ & 12.0 \\
\hline 10 & July 31,1983 & $35^{\circ} 59.04^{\prime}$ & $-117^{0} 50.91^{\prime}$ & 7.4 \\
\hline 11 & April 6, 1984 & $35^{\circ} 21.62^{\prime}$ & $-117^{\circ} 54.30^{\prime}$ & 10.2 \\
\hline 12 & Sept. 7, 1983 & $35^{\circ} 43.07^{\prime}$ & $-118^{\circ} 03.63^{\prime}$ & 7.0 \\
\hline 13 & April 11, 1984 & $35^{\circ} 47.31^{\prime}$ & $-118^{\circ} 02.21^{\prime}$ & 9.4 \\
\hline 14 & April 12,1984 & $35^{\circ} 45.51^{\prime}$ & $-117^{\circ} 59.71^{\prime}$ & 7.7 \\
\hline 15 & Aug. 16,1980 & $36^{\circ} 11.31^{\prime}$ & $-117^{0} 53.11^{\prime}$ & 9.6 \\
\hline 16 & Aug. 22,1980 & $35^{\circ} 54.18^{\prime}$ & $-117^{\circ} 43.56^{\prime}$ & 9.5 \\
\hline
\end{tabular}

The attenuation anomaly is established by events not. only in the Brawley area but also outside the area (for example, event 9 on Figure $5 a$ ). The same block size as in the Coso-Indian Wells study was used ( $2 \mathrm{~km} \times 2 \mathrm{~km} \times 0.2 \mathrm{~km})$. The model was divided into 80 blocks across (E-W) and 72 blocks in the N-S direction, covering an area of $160 \mathrm{~km} \mathrm{x}$ $144 \mathrm{~km}$. The model had 80 blocks in depth, extending to $16 \mathrm{~km}$. The results were averaged over several layers and presented in 4-km-thick depth slices.

In analyzing the data, amplitudes of $P$ waves were determined by measuring the maximum peak-to-peak amplitudes on the seismogram at the expected $P$ arrival time. The $S$ wave amplitudes were also maximum peak-to-peak amplitudes within a 0.5 -s time window before or after the expected arrival time of direct $P$ and $S$ waves, or $P g$ and $S g$ waves. On some seismograms the $S$ wave period is longer than the $P$ wave period, suggesting some attenuation of high-frequency $S$ waves, though this could be a source effect. The values $R_{\theta} / R_{p}$ were determined by estimating the focal mechanism for each earthquake from first motion data (Figures $4 b$ and $5 b$ ).

\section{RESULTS FOR THE COSO REGION}

We first used a homogeneous half-space to invert the data and compared the results with those obtained by the screening technique described by Sanders [1984]. Figure 6 shows the results from the half-space model inversion for the Coso region at depth ranges of $1-3,3-5$, and $5-7 \mathrm{~km}$. Results are shown in terms of $1 / Q$ such that solid circles indicate high attenuation (low $Q$ ). The larger the size of the circles, the stronger the attenuation. Smallest circles indicate infinite $Q$. The term $k_{1} k_{2}$ is assumed 1 in the half-space inversion. The main purpose of the half-space experiment is to compare the geometry of the anomalous bodies obtained by the half-space inversion with that inferred from the observations of screening. The exact value of $k_{1} k_{2}$ is not important for this comparison. Locations of anomalous attenuating bodies inferred from screening of $S$ waves [Sanders et al., 1988] are plotted on the same figures for each depth range. The anomalies mapped by the half-space inversion correspond closely to those obtained by screening.

We next used the vertically varying structure shown in

TABLE 1b. List of Dates and Hypocenters of 15 Earthquakes Used in the Imperial Valley Study

\begin{tabular}{|c|c|c|c|c|}
\hline$\overline{\text { Event }}$ & Date & Latitude & Longitude & Depth,km \\
\hline 1 & Dec. 24, 1983 & $32^{\circ} 58.22^{\prime}$ & $-115^{\circ} 33.85^{\prime}$ & 8.62 \\
\hline 2 & Sept. 2, 1983 & $33^{\circ} 18.44^{\prime}$ & $-116^{\circ} 20.55^{\prime}$ & 8.95 \\
\hline 3 & Nov. 15,1983 & $33^{\circ} 02.10^{\prime}$ & $-115^{\circ} 33.67^{\prime}$ & 8.77 \\
\hline 4 & Oct. 15,1983 & $32^{\circ} 57.43^{\prime}$ & $-115^{\circ} 30.12^{\prime}$ & 9.43 \\
\hline 5 & Dec. 12,1983 & $33^{\circ} 03.74^{\prime}$ & $-116^{\circ} 03.22^{\prime}$ & 8.28 \\
\hline 6 & Jan. 30, 1984 & $33^{\circ} 02.72^{\prime}$ & $-116^{\circ} 12.70^{\prime}$ & 2.49 \\
\hline 7 & April 26,1984 & $32^{\circ} 44.77^{\prime}$ & $-115^{\circ} 25.92^{\prime}$ & 11.88 \\
\hline 8 & April 22, 1984 & $32^{\circ} 49.14^{\prime}$ & $-115^{\circ} 26.73^{\prime}$ & 9.87 \\
\hline 9 & Oct. 18,1984 & $32^{\circ} 49.85^{\prime}$ & $-115^{\circ} 36.96^{\prime}$ & 10.56 \\
\hline 10 & Oct. 3, 1984 & $32^{\circ} 48.60^{\prime}$ & $-115^{\circ} 42.96^{\prime}$ & 10.11 \\
\hline 11 & April 17, 1984 & $32^{\circ} 56.96^{\prime}$ & $-115^{\circ} 51.99^{\prime}$ & 8.13 \\
\hline 12 & May 29, 1984 & $32^{\circ} 52.16^{\prime}$ & $-115^{\circ} 31.73^{\prime}$ & 8.26 \\
\hline 13 & March 21, 1984 & $32^{\circ} 58.86^{\prime}$ & $-115^{\circ} 32.75^{\prime}$ & 9.12 \\
\hline 14 & April 29, 1984 & $32^{\circ} 58.04^{\prime}$ & $-115^{\circ} 31.62^{\prime}$ & 9.26 \\
\hline 15 & Oct. 30,1984 & $32^{\circ} 56.51^{\prime}$ & $-115^{\circ} 32.79^{\prime}$ & 10.18 \\
\hline
\end{tabular}




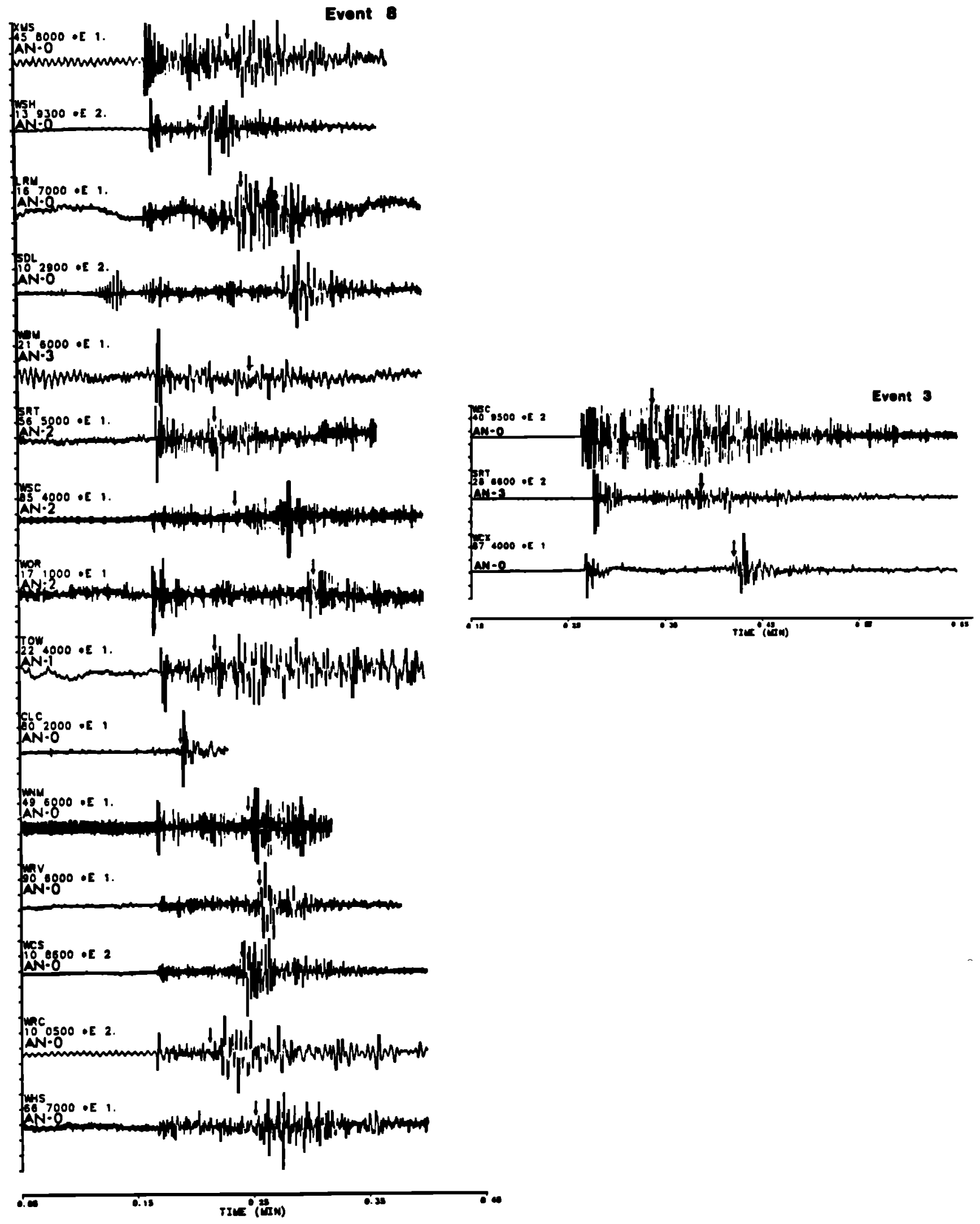

Fig. 4a. Examples of seismograms in the Coso-Indian Wells region with attenuated $S$ arrivals. Arrows indicate the expected S arriva] time [Sanders et al., 1988]. 

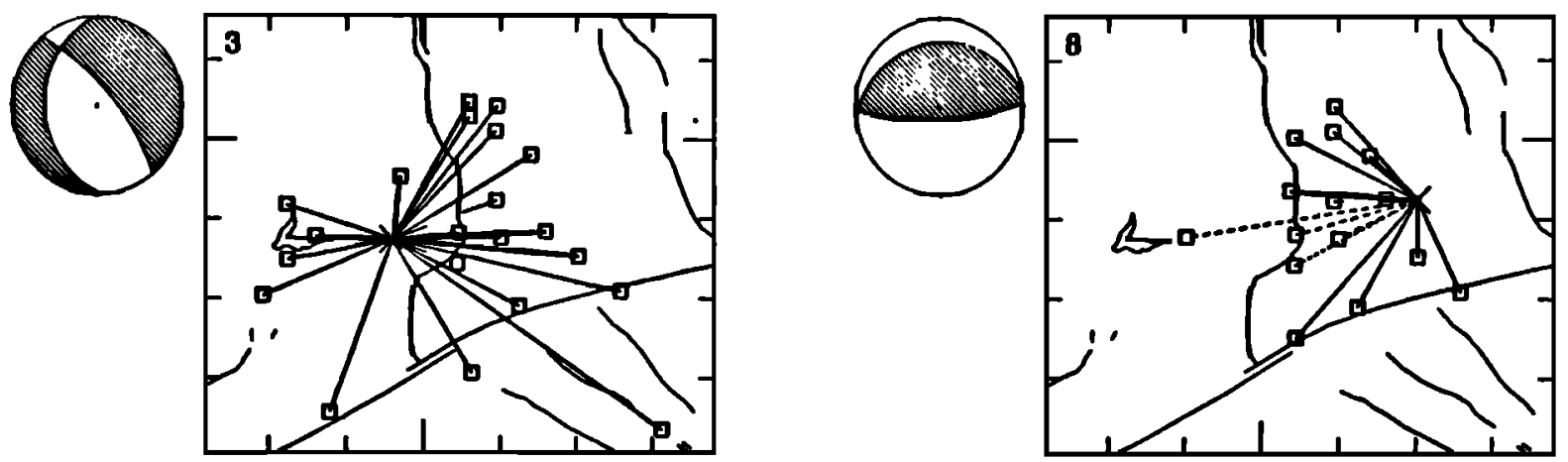

Fig. 4b. Plots of ray paths with indication of degree of attenuation for the two events shown on Figure 4a. Short dashes are for highly attenuated rays; long dashes are for partially attenuated rays and solid lines are for normal rays [Sanders et al., 1988]. The focal mechanisms are also plotted on the top left corner of each event.

Figure $7 a$ for lnversion. This structure was obtained by Walter and Weaver [1980] and was used by Walck and Clayton [1987] for velocity inversion. Results with $k_{1} k_{2}=$ 1 are shown in Figure 8a. Depth slices from $P$ wave travel time tomography [Walck and Cleyton, 1987] are shown in Figure 8b. Since the travel time inversion was done using a larger data set of more than $\mathbf{4 0 0}$ earthquakes, we did not perform travel time inversion using the smaller data set used for attenuation tomography. There is a good correlation between low-velocity anomalies and high-attenuation anomalies, despite the fact that a smaller data set was used in the latter. Although the data set used in the amplitude inversion was relatively small, the ray coverage in the central region was dense at depths from 3 to $9 \mathrm{~km}$, as indi-

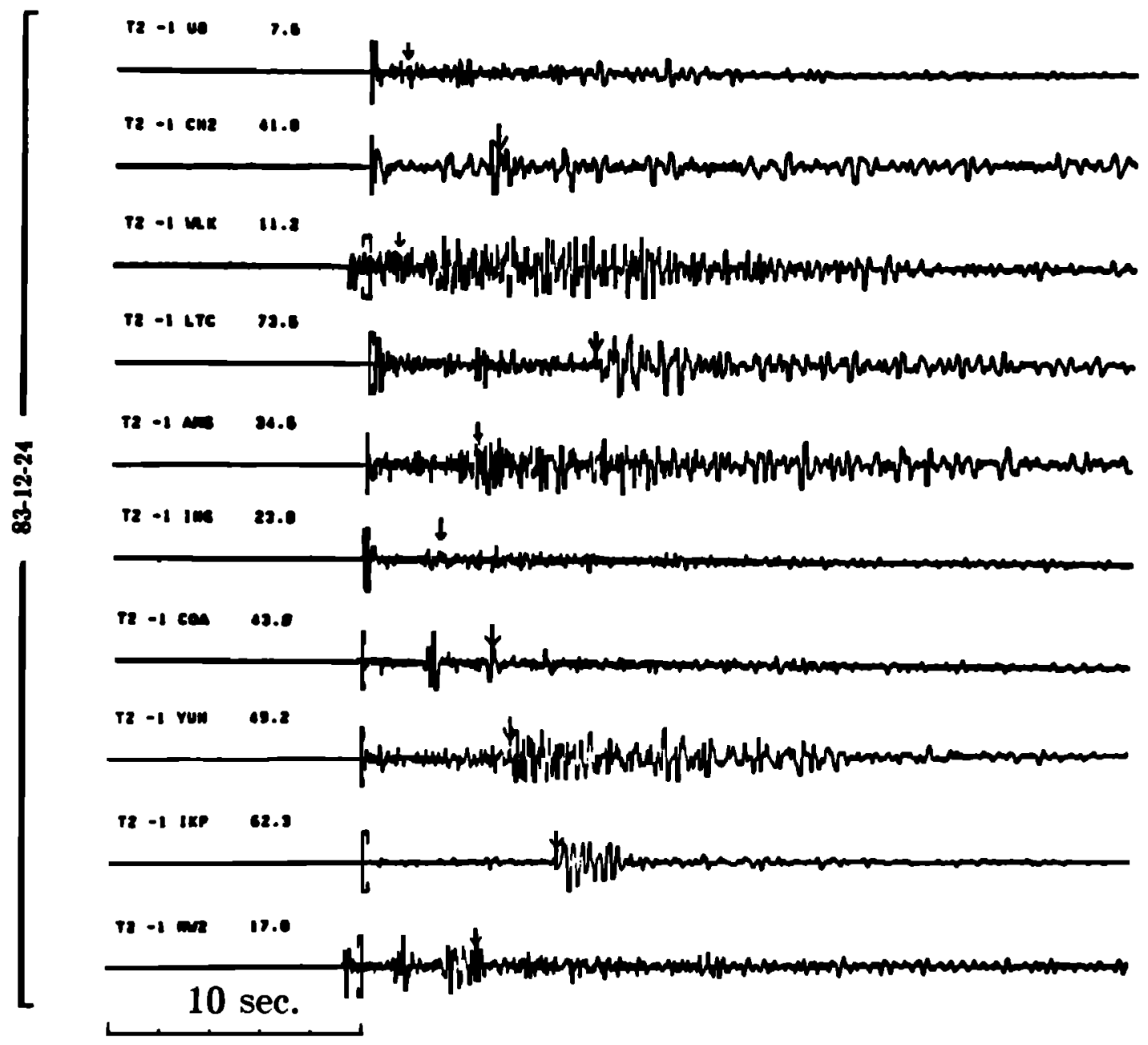

Fig. 5a. Examples of seismograms in the Imperial Valley with attenuated $S$ arrivals. Arrows indicate the expected $S$ arrival time. 


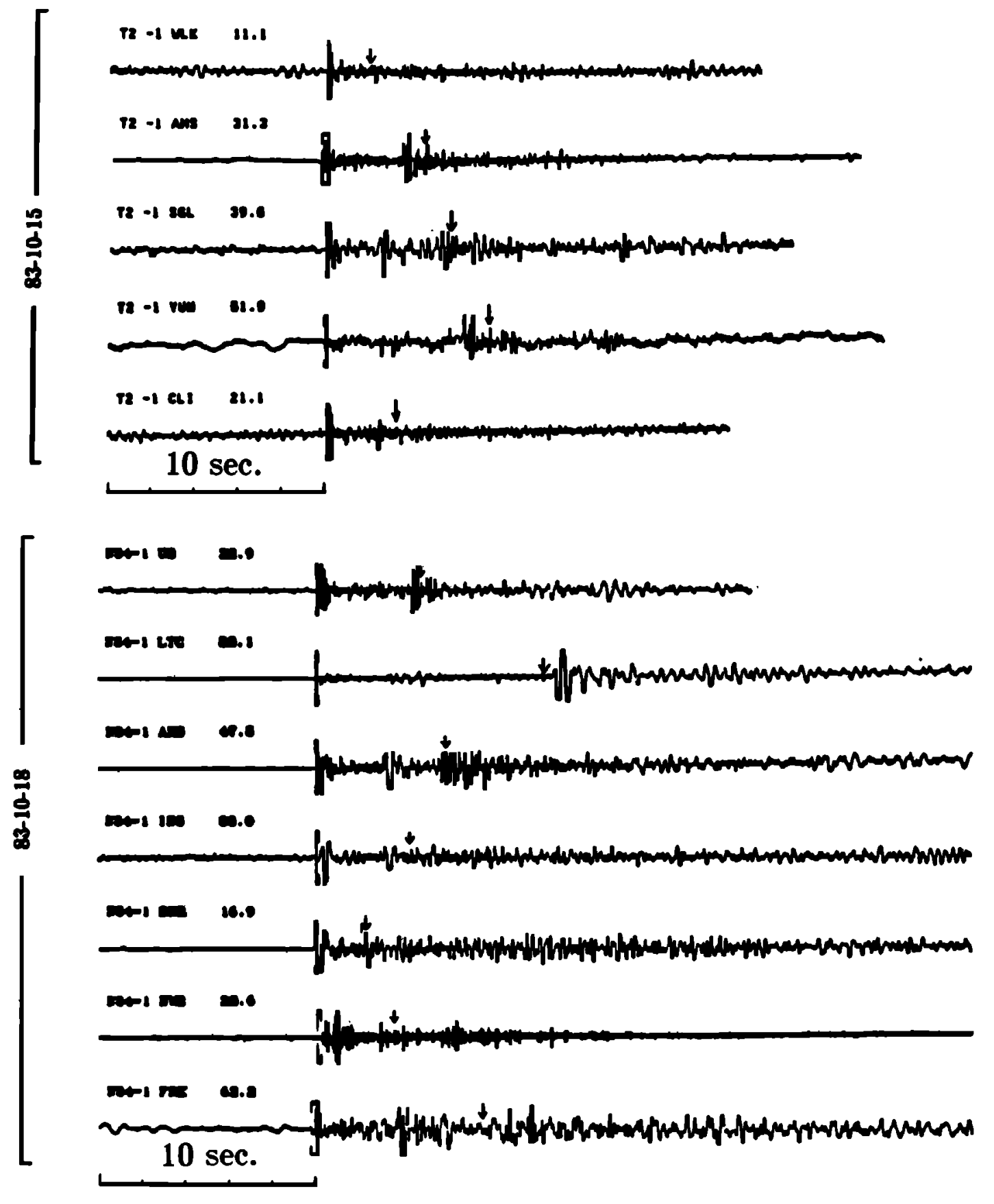

Fig. 5a. (continued):

cated by the large number of hit counts shown in Figure $8 c$. The number of rays used in both cases was of the order of 300 . We inverted the data for blocks with two hit counts or more. We also inverted the data for blocks with four or more hit counts. Since the blocks with four or more hit counts are relatively sparse, the results were given only for these sparse blocks. However, if these sparse anomalies were spatially smoothed, the overall pattern was essentially the same as that obtained for blocks with two or more hit counts. These anomalies include the major anomalies in both Indian Wells Valley and Brawley areas (see next section for results on the Imperial Valley). For layers with sparse ray coverage, for example, layers 1 and 2 in Coso region and layer 1 in Imperial Valley, any anomalies are considered insignificant. Since this tomographic inversion used the number of hit counts of a block as the weighting factor for that block, the higher the number of hit counts, the more reliable the result is. There is an apparent correspondence between the locations of resolved anomalies and dense hit counts (Figures $8 a$ and $8 c$ and Figures 11 and 12a), suggesting the possibility that the resolved anomalies are artifacts of the dense hit counts due to the weighting scheme used in this method. In order to examine this, we used a generalized inversion technique (P. Ho-Liu et al., 


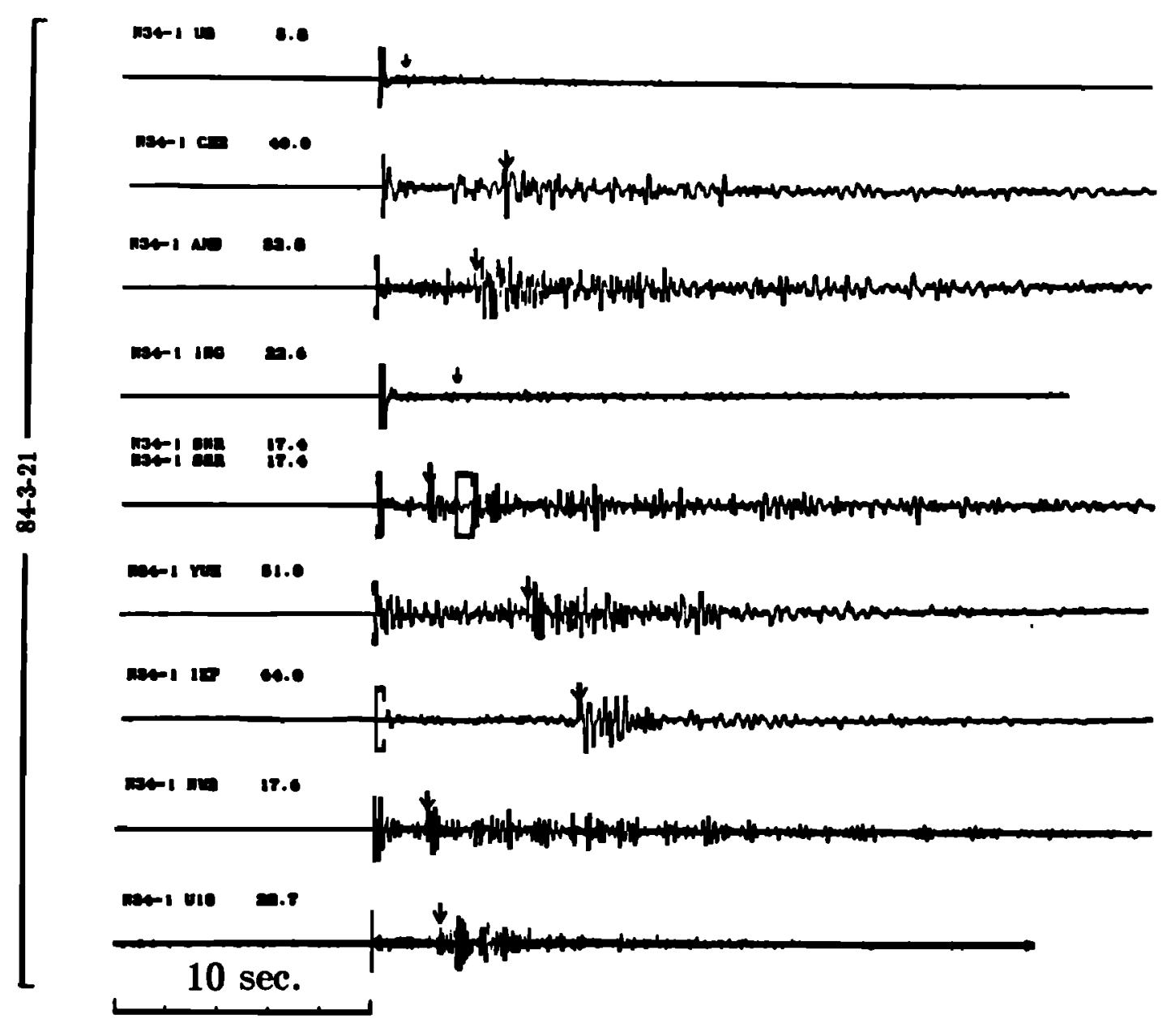

Fig. 5a. (continued)
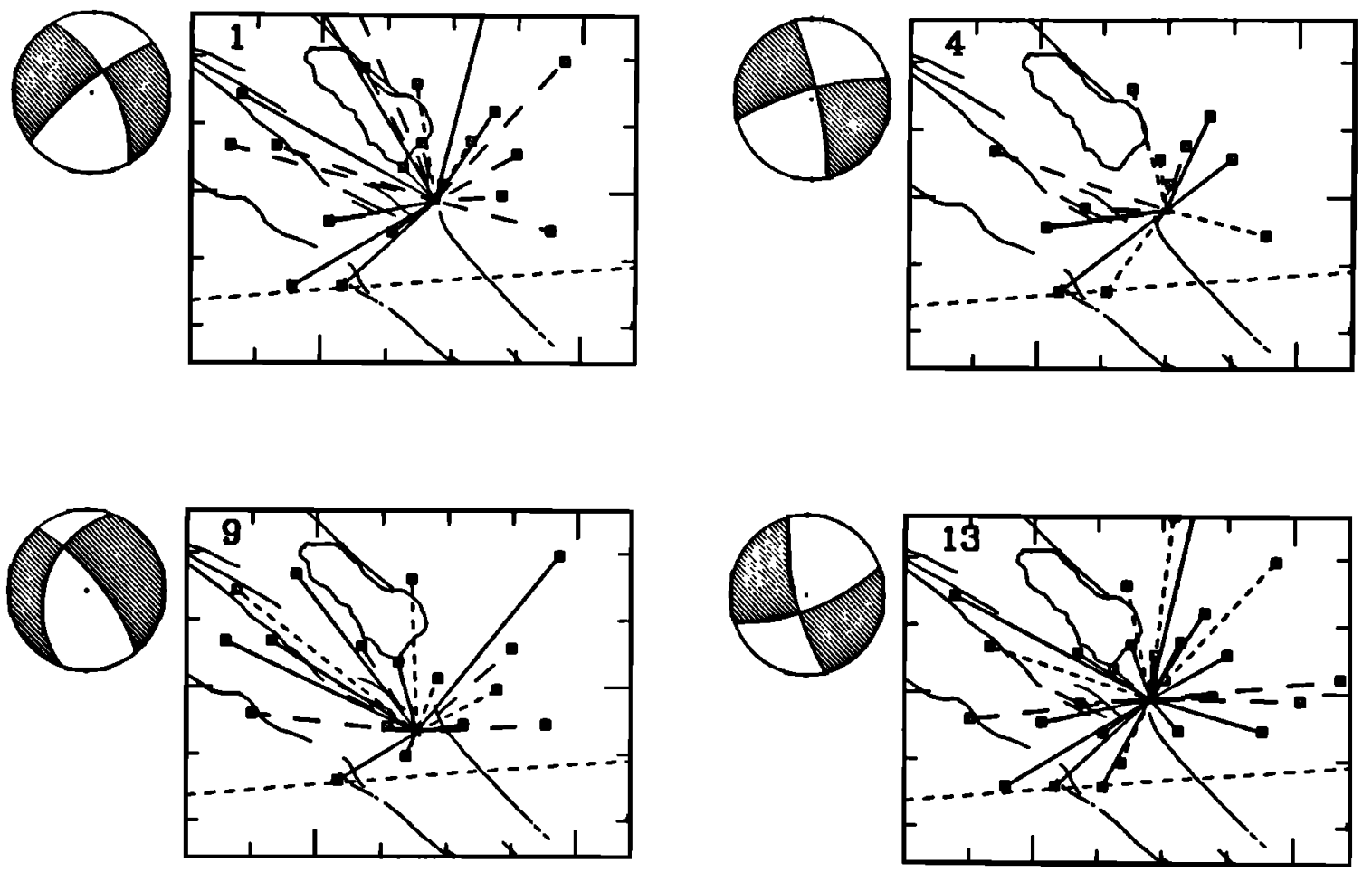

Fig. 5b. Plots of ray paths with indication of degree of attenuation for the four events shown on Figure $5 a$. Degree of attenuation is indicated by the types of lines as indicated as in Figure $4 b$. The focal mechanisms are also plotted on the top left corner of each event. 


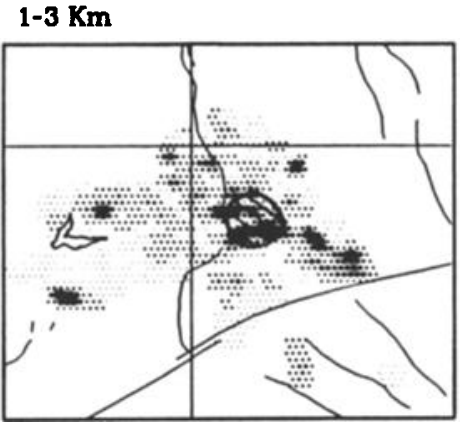

3-5 Km

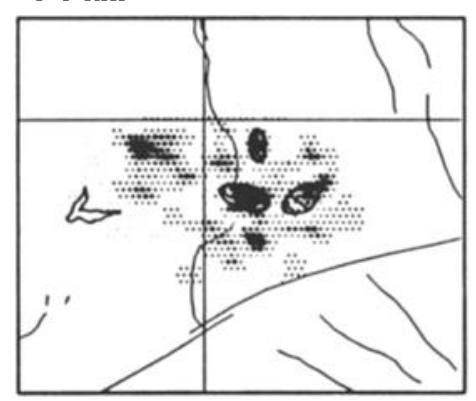

$40 \mathrm{~km}$
5-7 Km

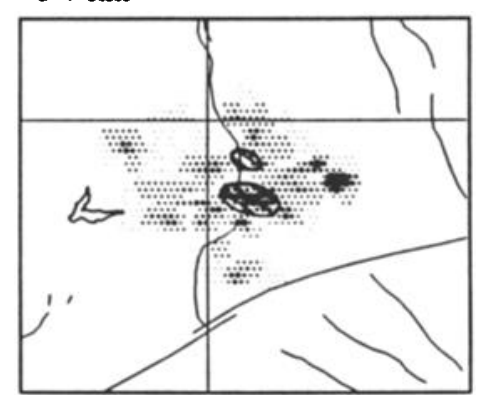

Coso region: Halfspace Attenuation Inversion

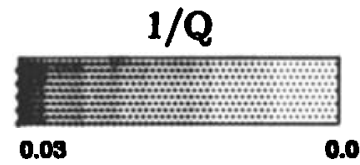

0.0

Fig. 6. Results of half-space inversion for Coso region. Depth slices at the depths of 1-3, 3-5, and 5-7 km are shown, together with the anomalies located by the forward modeling of Sanders et al. [1988]. Solid circles indicate areas with high attenuation, and open circles indicate areas with low attenuation. Cross-hatched areas are the resolved anomalies from the forward modeling of Sanders et al. [1988). Locations of the anomalies in each depth slice obtained by inversion and forward modeling agree well.

unpublished manuscript, 1987) which uses an entirely different approach to test the resolution. We found that the locations of the anomalies are well resolved in both cases. Therefore we conclude that these resolved anomalies are not artifacts of the weighting scheme used in the back projection method. The quality factor $Q_{\beta}$ in the attenuat- ing body is as low as 30 , and the average $Q_{\beta}$ in the whole region is about 160 .

In view of the uncertainty in $k_{1} k_{2}$, we inverted the same data with $k_{1} k_{2}=10$ (Figure $8 d$ ) and a value of $k_{1} k_{2}=0.5$ (Figure $8 e$ ). Although the spatial extent of the anomalous body in the depth range $3-5 \mathrm{~km}$ is slightly reduced in both
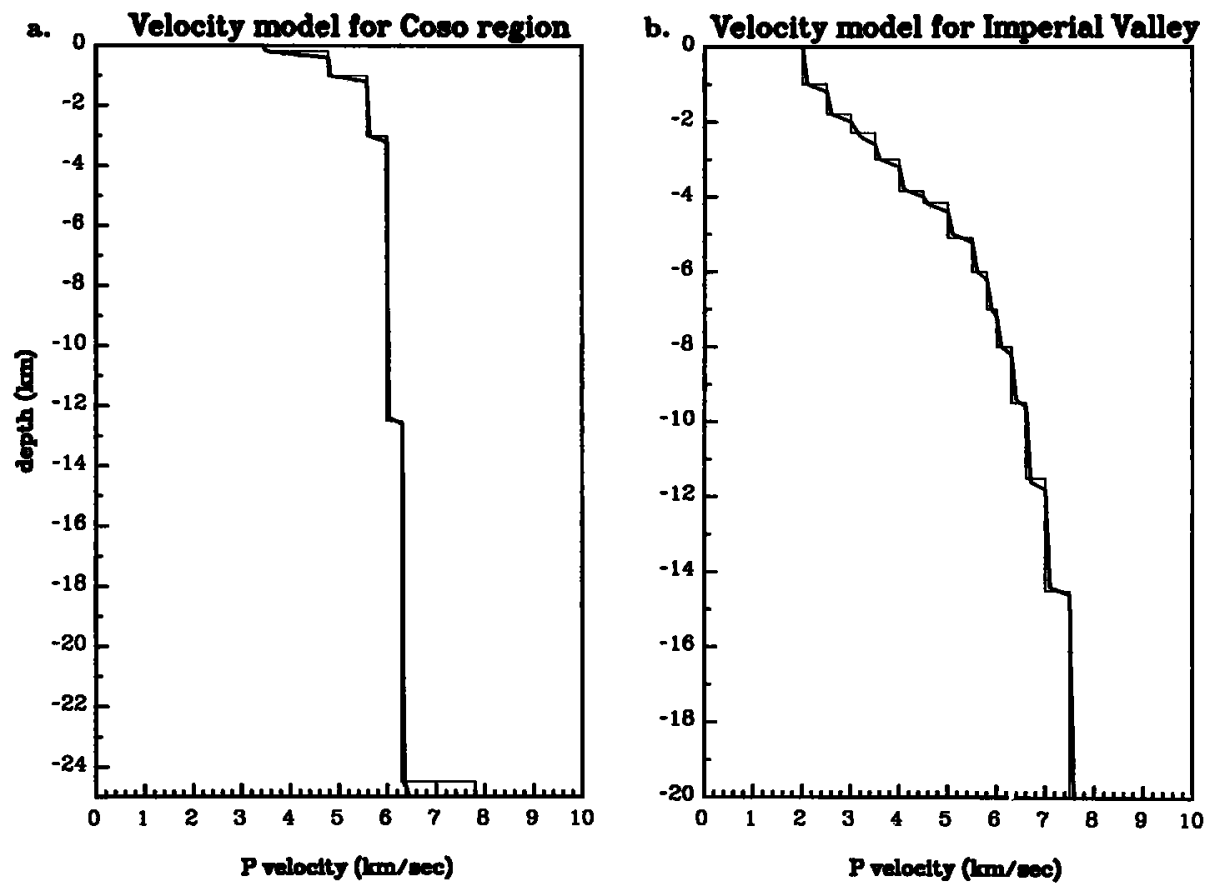

Fig. 7. (a). Velocity-depth function used in Coso region study. Thin line indicates the velocity model by Walter and Weaver [1980) and bold line is the modified velocity model actually used to trace rays. (b). Velocity depth function used in Imperial Valley study. Thin line indicates the velocity model of $F$ uis et al. [1982] and bold line is the modifled velocity model used to trace rays. 
$0-1 \mathrm{Km}$

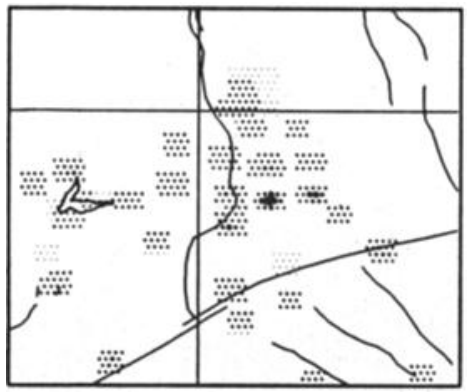

$5-7 \mathrm{Km}$

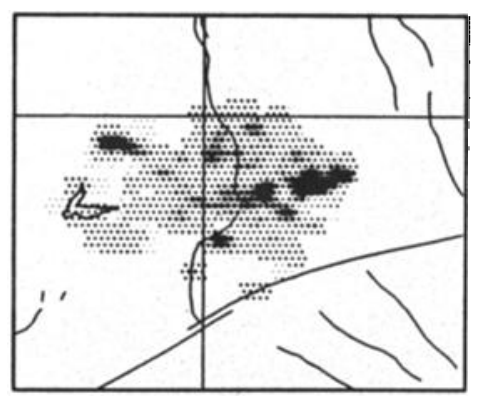

$1-3 \mathrm{Km}$

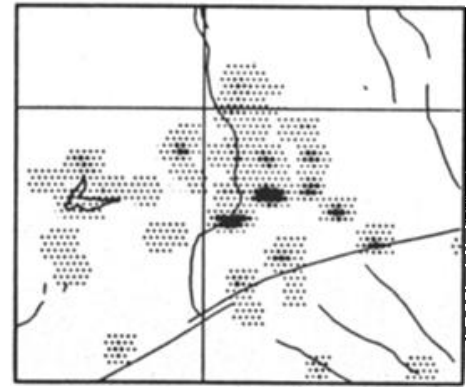

7-8 km

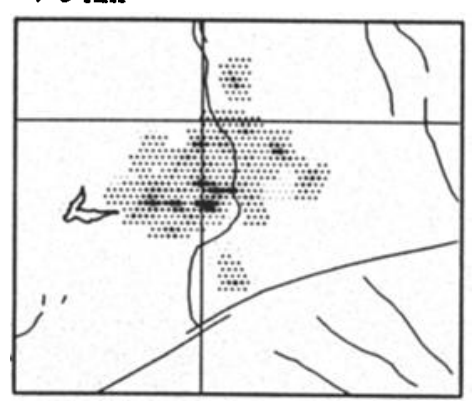

3-5 Km

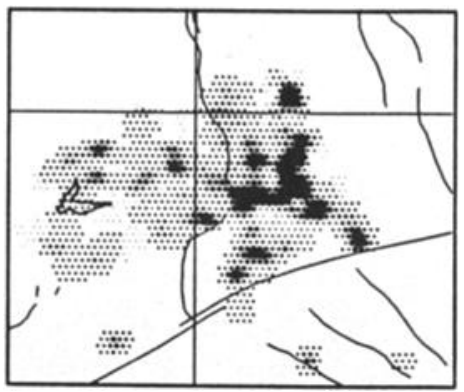

Coso Region: Attenuation Inversion Q1

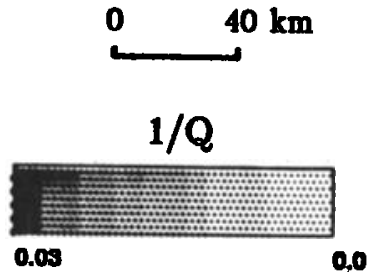

Fig. 8a. Results of attenuation inversion using the Coso velocity model shown in Figure 2a. Depth slices at (1) 1-3 $\mathrm{km}$, (2) $3.5 \mathrm{~km}$, (3) $5-7 \mathrm{~km}$, and (4) 7-9 $\mathrm{km}$ are shown. A value of 1 was used for $k_{1} k_{2}$. Attenuation anomalies are resolved beneath the Coso Range and the Indian Wells Valley in the depth range of $3-5 \mathrm{~km}$. The locations of these two anomalies agree very well with the low-velocity anomalies obtained by inversion of $\boldsymbol{P}$ wave travel times (Walck and Clayion, 1987]. The anomalies at the edge of ray coverage (see Figure 8c) are probably spurious resulting from low hit counts or poor aximuthal ray coverage. The Coso range anomaly disappears below $5 \mathrm{~km}$, but the Indian Wells Valley anomaly still exists at 5-7 km depth range.
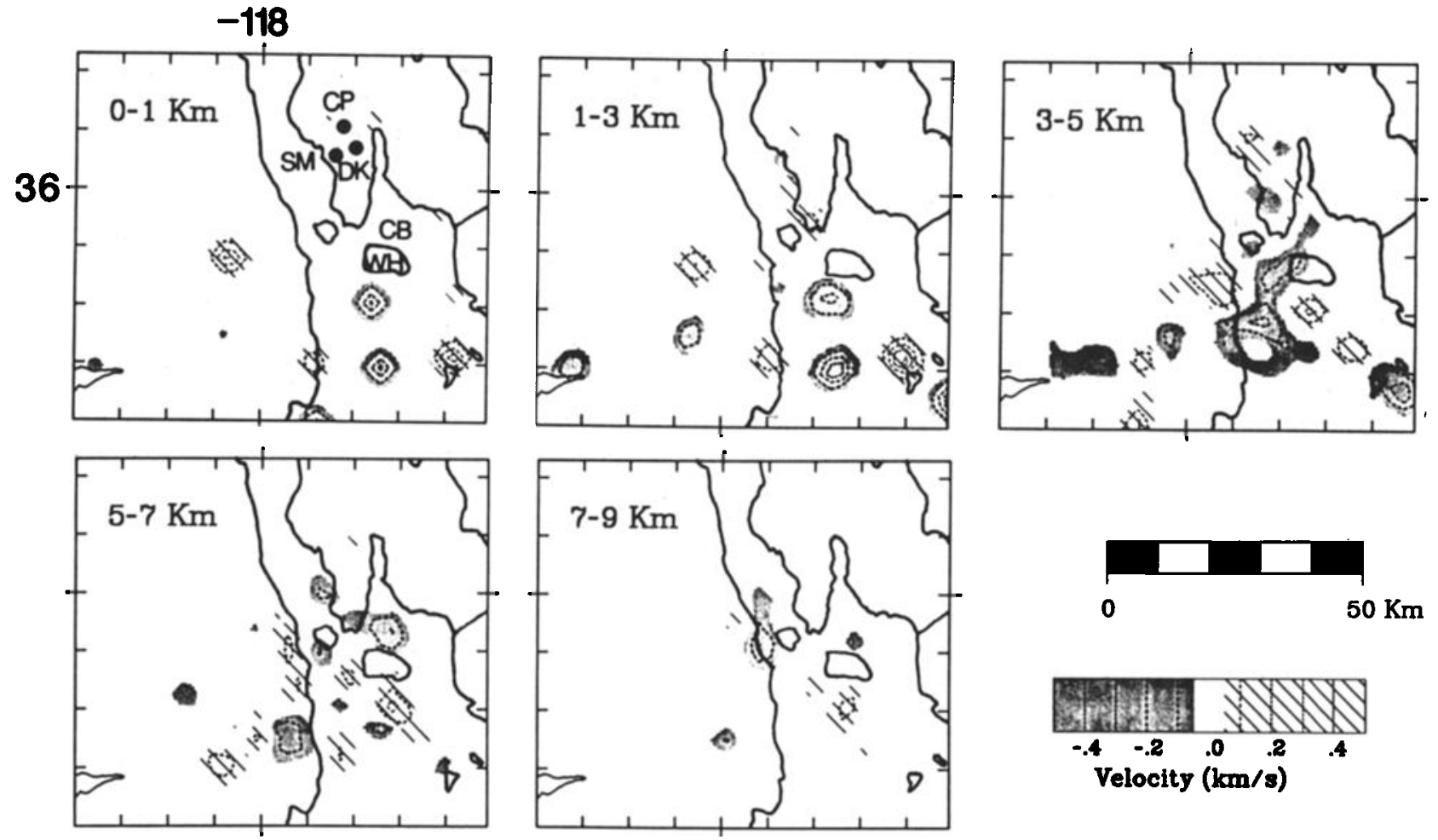

Fig. 8b. Results of travel time tomography [Walck and Clayton, 1987] for the same depth slices as in Figure 8a. The area covered by their study $(80 \mathrm{~km} \mathrm{x} 70 \mathrm{~km})$ is smaller than the area corered by this attenuation study $(144 \mathrm{~km} \times$ $144 \mathrm{~km}$ ). OP stands for Cactus Peak, SM for Sugarloar Mountain, CB for Coso Basin, DK for Devil's Kitchen, and WH for White Hills. Shaded regions indicate slow anomalies while hachured areas are fast. Notice the good agreement in locations of these two anomalies (Coso Range and Indian Wells Valley anomalies) in the depth slice of 3-5 $\mathrm{km}$ with the enomalies resolved by attenuation inversion shown in Figure $8 \mathrm{a}$. 
0-1 Km

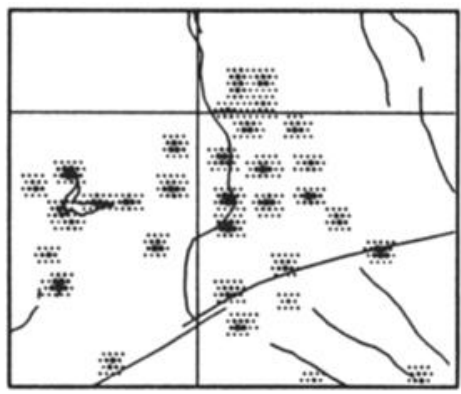

5-7 Km

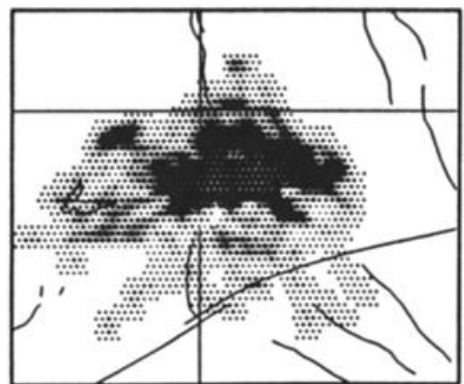

1-3 Km

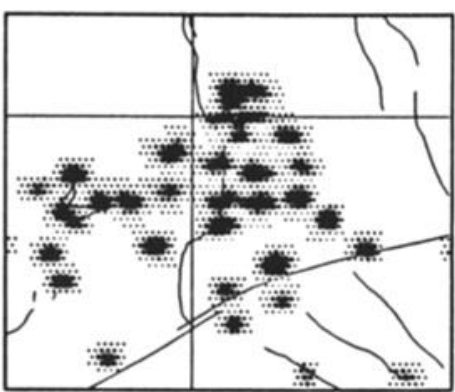

7-9 Km

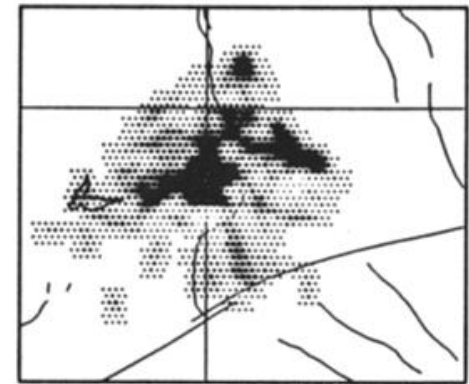

3-5 Km

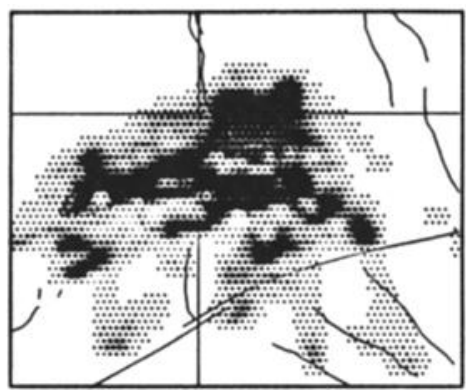

Coso region: Attenuation Hitcounts

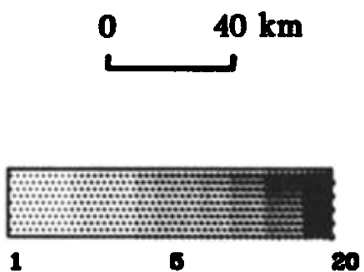

Fig. 8c. Hit count maps of Coso for the depth slices of (1) $1-3 \mathrm{~km},(2) 3-5 \mathrm{~km}$, (3) 5-7 km, and (4) 7-9 km. The depth slice of $3-5 \mathrm{~km}$ is well covered at the locations of the two resolved anomalies. The hit count is highest in the center of the model in both 3-5 km and 5-7 km depth slices.

cases, the overall pattern is the same as that shown in Figure $8 a$ (with $k_{1} k_{2}=1$ ). Because of the uncertainty in $k_{1} k_{2}$, the size and the $Q_{\rho}$ value of the attenuating body are not definitive, but the overall geometry of the attenuating bodies in the Indian Wells Valley appears to be well resolved.

\section{RESULTS FOR THE IMPERIAL VALLEY}

Figure 9 shows the results for the half-space model for the Imperial Valley together with the anomalies inferred by screening. The value of $k_{1} k_{2}$ is 1 in the half-space inversion. Attenuation anomalies are found in both the 3-5 and
$0-1 \mathrm{Km}$

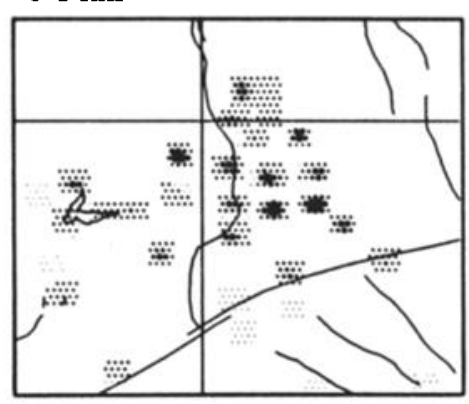

5-7 Km

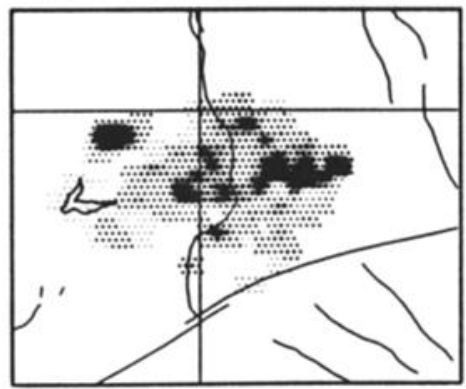

$1-3 \mathrm{Km}$

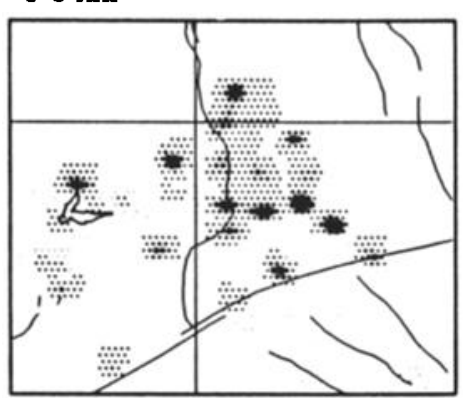

$7-9 \mathrm{Km}$

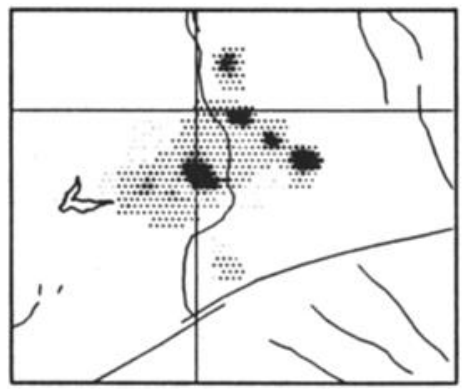

3-5 Km

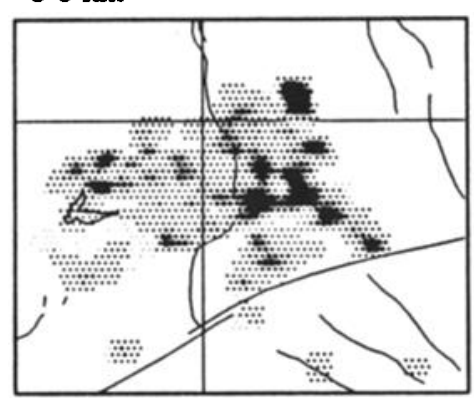

Coso region: Attenuation Inversion Q10

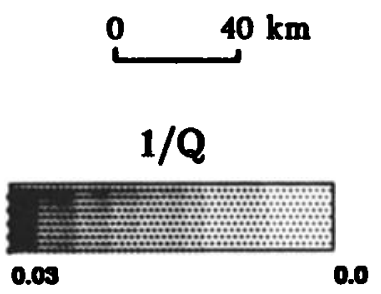

Fig. $8 d$ Results of attenuation inversion with $k_{1} k_{2}=10$. The two anomalies in the depth range of $3-5 \mathrm{~km}$ still exist, and the general pattern resembles that shown in Figure 8a. Anomalies at the edge of ray coverage (e.g., the anomaly just northeast of Lake Isabella in the depth range of 5-7 km) are probably spurious. The value of $Q_{\beta}$ remains approximately the same as in Figure $8 \mathrm{a}$. 

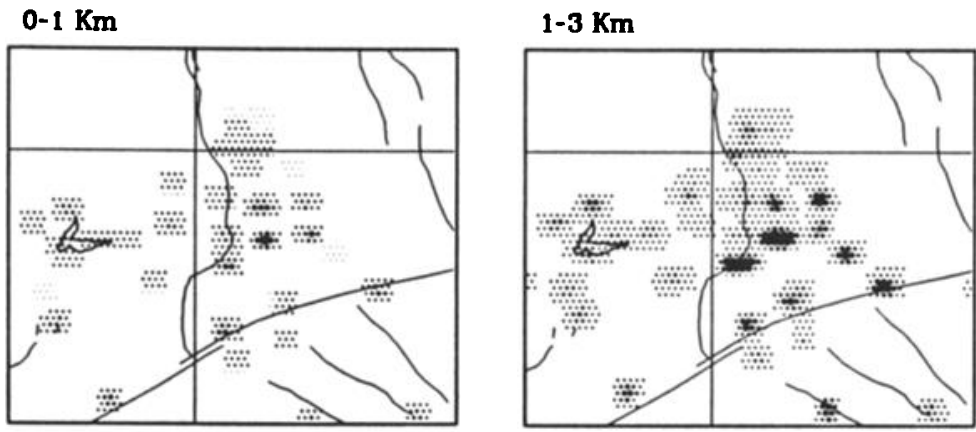

3-5 Km
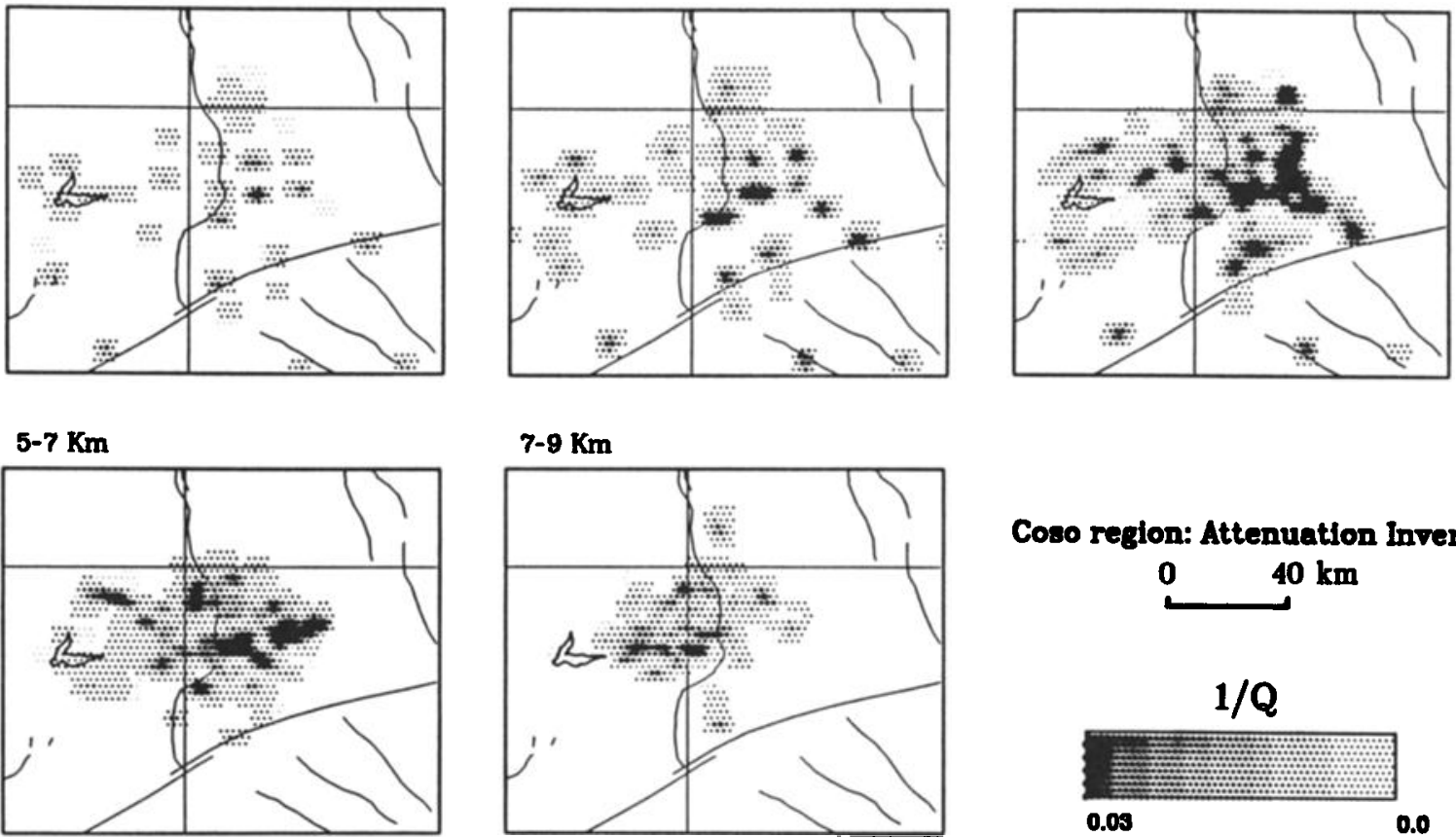

Coso region: Attenuation Inversion Q.5
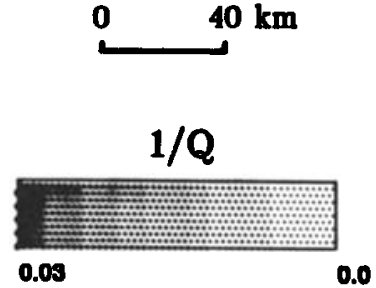

Fig. 8e. Results of attenuation inversion with $k_{1} k_{2}=0.5$. The Indian Wells Valley anomaly still exists in the range of 3-5 km. The image of the anomaly remains the same as in Figure 8a. The value of $Q_{\beta}$ is slightly lower than the value of the anomaly in Figure $8 a$ but is of the same order of 30 .

$5-8 \mathrm{~km}$ depth slices in the screening. The largest anomaly is located just north of the Imperial fault in the southern Brawley seismic zone in the $5-8 \mathrm{~km}$ depth slice. By inverting the amplitude data in a half-space model, we found anomalies in the $3-5$ and $5-8 \mathrm{~km}$ depth slices at almost the same locations as from screening.

Next, we relocated all earthquakes used in the analysis (Figure 3) using HYPO71 [Lee and Lahr, 1975] and traced the rays through the velocity model derived by $F$ uis et al. [1982] for the southern Imperial Valley (Figure 7b).

The result of travel time tomography is shown in Figure 10. A slow anomaly is found north of the Imperial fault in the Brawley seismic zone. The anomaly is deeper than that found by using the half-space model because rays are traveling downward from the earthquakes to the stations in this velocity model. In the half-space model the rays travel

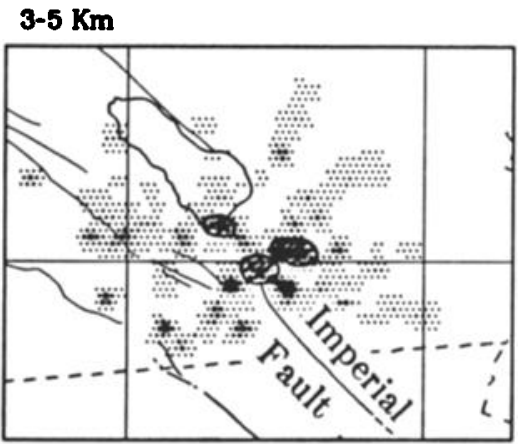

5-8 Km

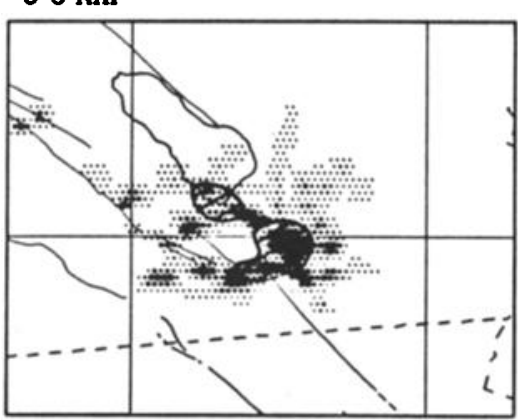

$40 \mathrm{~km}$

Imperial Valley: Halfspace Attenuation Inversion

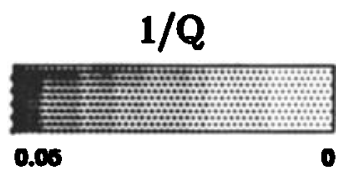

Fig. 9. Results of half-apace inversion for Imperial Valley. Depth slices of 3-5 and 5-8 km are shown, together with the anomalies resolred by forward modeling ( $S$ wave screening technique). The major anomaly is that north of the Imperial fault in the southern portion of the Brawley seismic zone, in the depth range of 5-8 $\mathrm{km}$. The locations of the anomalies obtained by inversion and forward modeling agree well with each other. 

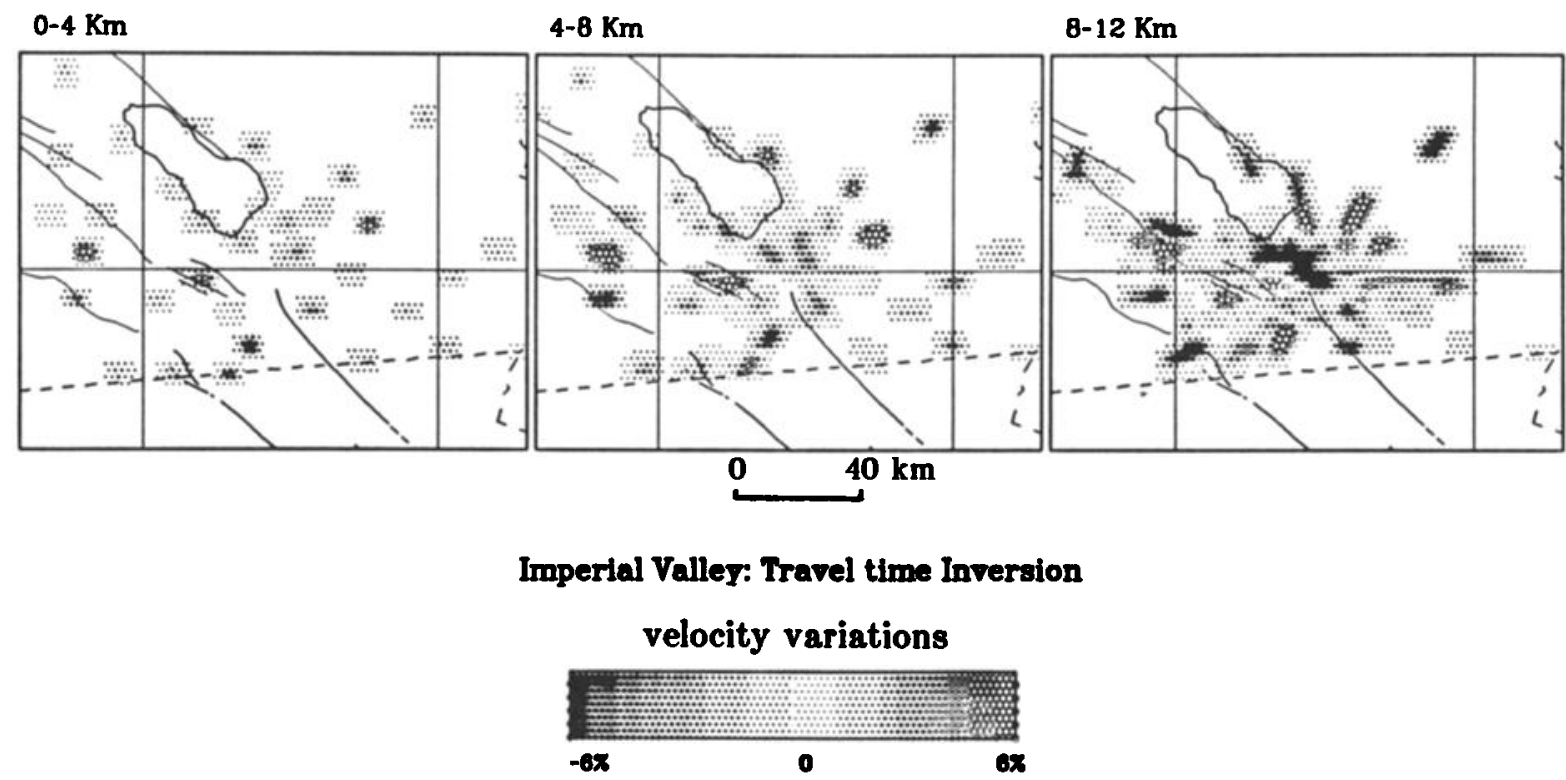

Fig. 10. Results of travel time inversion for the depth slices of (1) 0-4 km, (2) 4-8 km, and (3) 8-12 km. The scale indicates percentage variations in $P$ wave velocities. Solid circles indicate slow velocities, while open circles indicate fast velocities. The $8-12 \mathrm{~km}$ depth slice is best resolved. The velocity is $10 \%$ slower in the southern Brawley seismic zone in this depth range compared to the surrounding region.

straight up from the earthquakes, and the image of the anomaly is formed at a shallower depth. The velocity is about $10 \%$ slower than normal in the $8-12 \mathrm{~km}$ depth range. The number of hit counts is the highest in the area north of the Imperial fault in the depth range of $8-12 \mathrm{~km}$ (see Figure 12 for the hit count map of attenuation inversion). The hit count map of travel-time inversion is similar to Figure 11. With a larger number of hit counts in the Brawley seismic zone the weighting factor is higher, and therefore the results obtained in the Brawley seismic zone are more reliable than those in other areas of the model.
Figure $12 a$ shows the results of the attenuation tomography using the same data set with $k_{1} k_{2}=1$. A highly attenuating anomaly is located north of the Imperial fault in the vicinity of the slow velocity anomaly at the same depth range of 8-12 km. Figures $12 b$ and $12 c$ show the cesults of the attenuation tomography with $k_{1} k_{2}=10$ and $k_{1} k_{2}=0.5$, respectively. Notice that in both cases where $k_{1} k_{2}$ is not 1 , a highly attenuating anomaly is imaged in the southern Brawley seismic zone at 8-12 km depth. The value of $Q_{\beta}$ remains of the order of 20 in all three cases. This similarity suggests that the overall geometry of the

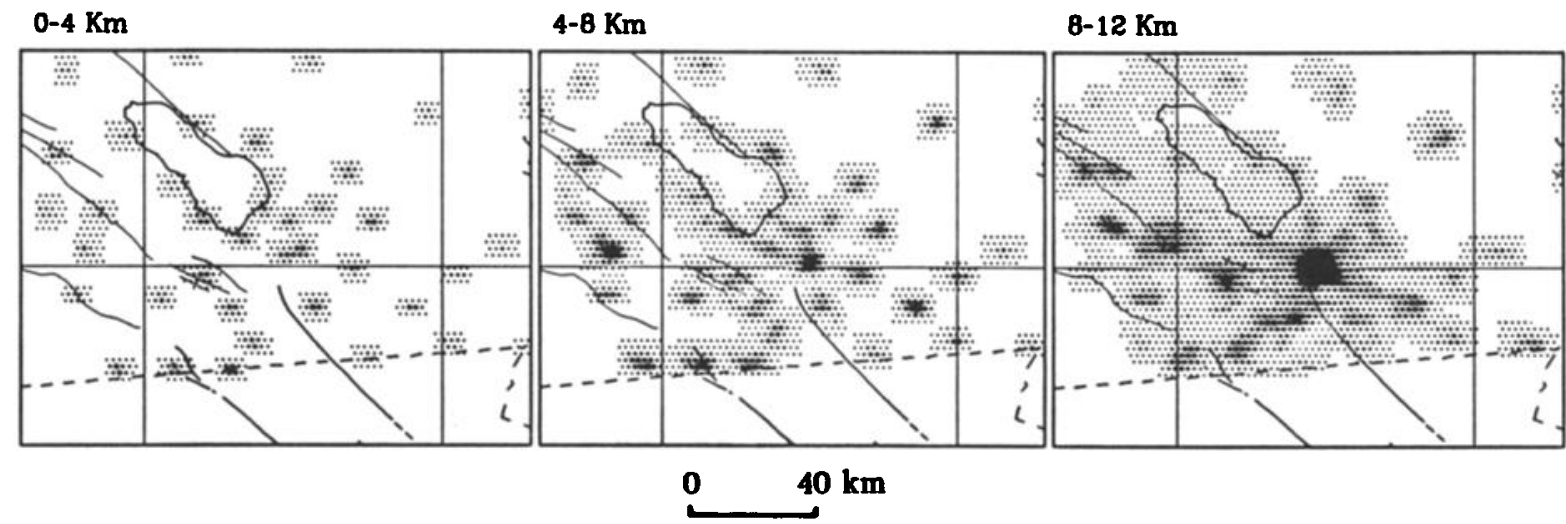

Imperial Valley: Attenuation Hitcounts

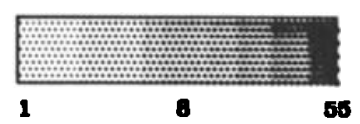

Fig. 11. Hit count maps for attenuation inversion in the same depth slices as in Figure 12. Travel time hit count distribution was approximately the same. The highest hit count occurs in the depth range of 8-12 km in the southern Brawley seismic zone. Any anomaly found in this depth range in the Brawley seismic zone is considered well resolved because of the high hit count. 
a). 0-4 Km 4-8 Km 8-12 Km
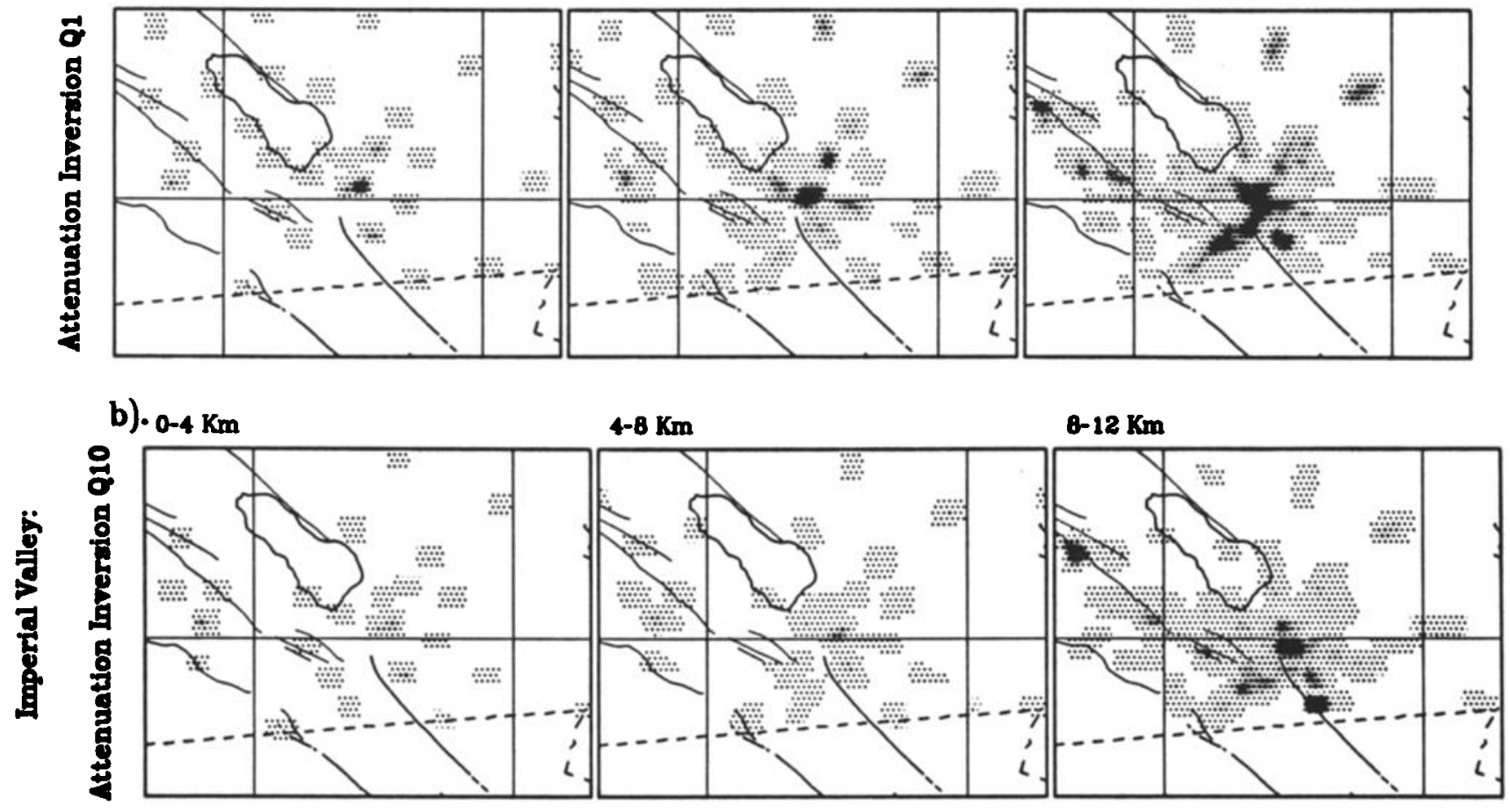

c). $0-4 \mathrm{Km}$

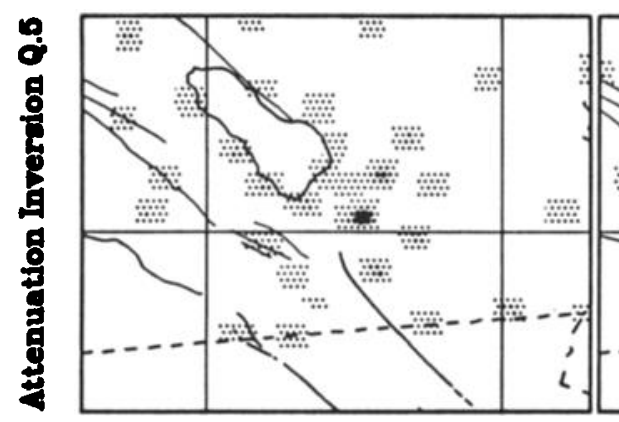

$4-8 \mathrm{Km}$

8-12 $\mathrm{km}$
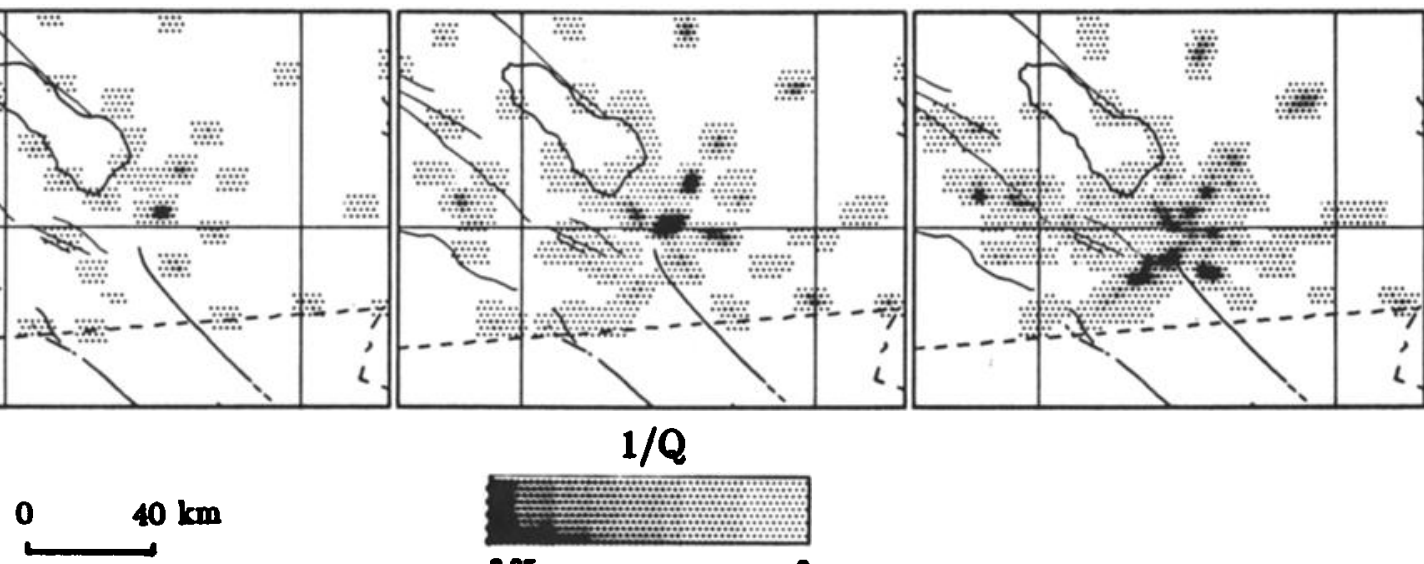

$1 / Q$

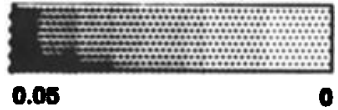

Fig. 12. (a) Results of attenuation inversion with $k_{1} k_{2}=1$. The best resolved depth slice of $8-12 \mathrm{~km}$ shows a highly attenuating anomaly in the southern Brawley seismic zone, correlated well with the slow anomaly from travel time inversion (Figure 10). (b) Results from attenuation inversion with $k_{1} k_{2}=10$. The attenuating anomaly is more concentrated in the general Brawley seismic zone. (c) Depth slices for results of attenuation inversion with $k_{1} k_{2}=0.6$. The attenuation anomaly is less intense in the Brawley seismic zone but the general geometry is the same in Figure 12a.

anomalous structure is resolved well despite the uncertainty in $k_{1} k_{2}$. This is not surprising because the distribution of the attenuated paths is essentially controlled by the locations of the attenuating bodies. The details of the structure, however, depend on the value of $k_{1} k_{2}$. Here we take the result with $k_{1} k_{2}=1$ as a representative structure. The average $Q_{\beta}$ for the area covered by the rays is about 140 which reflects the high attenuation due to soft sediments in the valley, a result similar to that obtained in an independent study by Lis [1983] on the 1976 Brawley earthquake.

\section{RESOLUTION EstmMATES AND NoISE EFTECTS}

In order to evaluate the resolution of our inversion we performed a test using synthetic data sets. We first generated a synthetic anomaly either at the location of an anomaly obtained by inversion of the data or at a location where azimuthal ray coverage is poor but the number of hit counts is high, as a test of the effect of poor azimuthal ray coverage on the results. We assigned $Q_{\beta}=1.0$ to one block at the center of either the anomaly obtained from real data or the area with poor azimuthal ray coverage, set- 
a). $7 \mathrm{Km}$

b).

\section{0-1 Km}

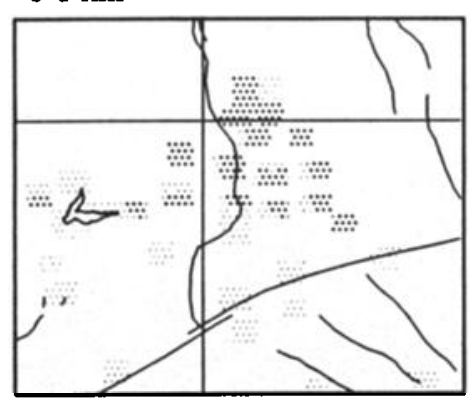

5-7 Km

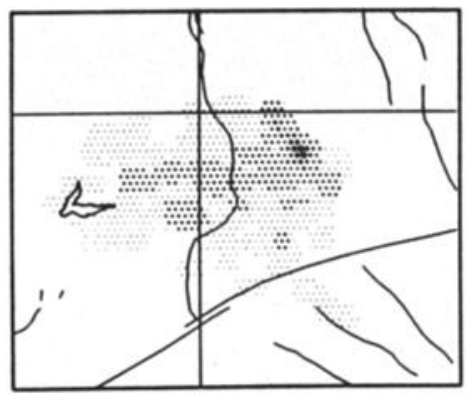

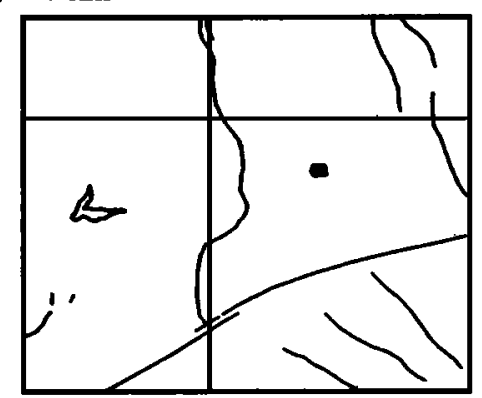

1-3 Km

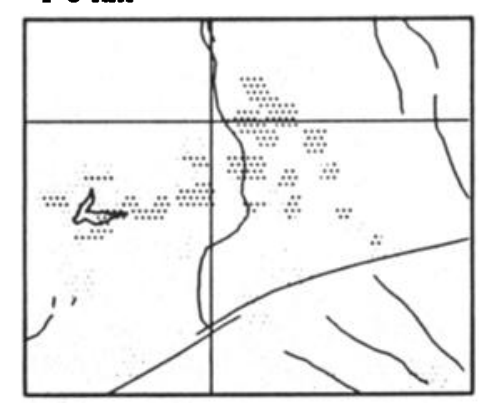

$7-9 \mathrm{Km}$

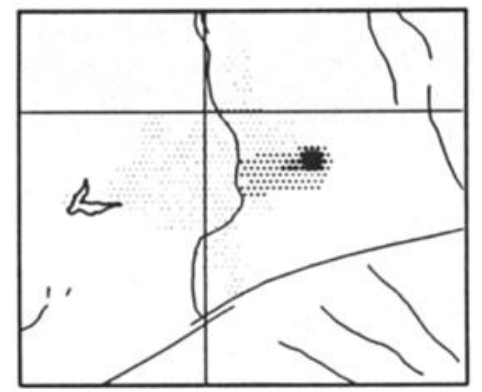

3-5 Km

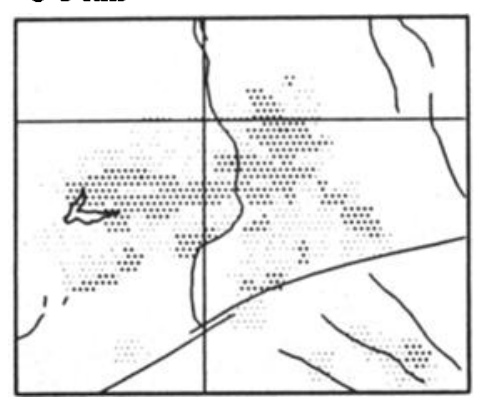

Coso region: Resolution test

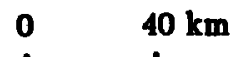

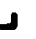

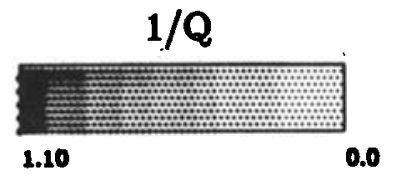

Fig. 13. (a) Location of a synthetic anomaly in the Coso study. A value of $Q_{\beta}=1.0$ was assigned to the shaded block. This block was chosen because of its high hit count but poor aximuthal ray coverage. The depth of this block is $7 \mathrm{~km}$. (b). Results of resolution test for the synthetic anomaly shown in Figure 13a. Northwestward ray steaking can be seen in the depth range $5-7 \mathrm{~km}$.

ting $Q_{\beta}$ to infinity elsewhere. Using this attenuation structure, we computed the amplitude for the rays used in the real data set. We inverted this synthetic data set to test the resolution of our inversion.

For the Coso ray set a synthetic anomaly with $Q_{\beta}=1.0$ is assigned to a block at $7 \mathrm{~km}$ depth with a high number of hit counts but situated at the edge of ray coverage as shown in Figure 13a. This block was of poor azimuthal coverage. As Figure 13b shows, some smearing of the image occurred to the west of the point anomaly. The smearing is more pronounced in the horizontal direction than in the vertical direction. About $90 \%$ of the anomaly was recovered by inversion after 30 iterations.

For the Imperial Valley a synthetic anomaly was generated for both the travel time set $(d s=1.0)$ and the amplitude set $\left(Q_{\beta}=1.0\right)$ and was given to the block at the center of the anomaly (Figure 14a) resolved by inversion of the actual data. This block was situated at a depth of 8.6 $\mathrm{km}$, and it carried the maximum hit count. Inversion results of travel time (Figure 146) and amplitude data (Fig- ure 14c) show some smearing of the image in the vertical or horizontal directions. After 30 iterations, about $83 \%$ of the travel time anomaly and about $97 \%$ of the attenuation anomaly were recovered.

In order to test the effects of noise on the inversion, the data set was replaced by random noise and was inverted. If the anomaly found by inversion of real data is caused by the presence of high noise level, inversion of random noise will image an anomaly at the same location as the anomaly found from inversion of the actual data. As shown in Figure 15, the synthetic data set consisting of random noise yielded a random distribution of anomalies at the depth slice where a single anomaly was found by inversion of real data. Therefore we believe that random noise was probably not a major cause for the anomalous structure for either Coso or Imperial Valley.

\section{Discussion}

Since most of the stations of the southern California network have only a vertical component seismometer, we had 
a).

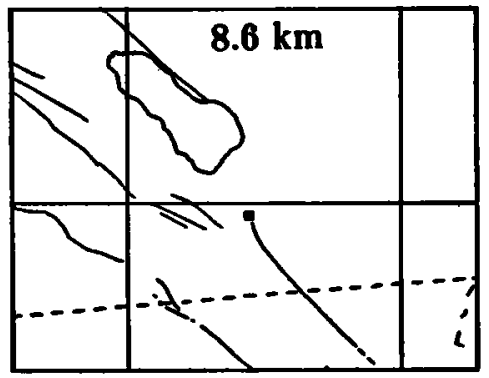

b). $0-4 \mathrm{Km}$

4-8 $\mathrm{km}$

6-12 Km
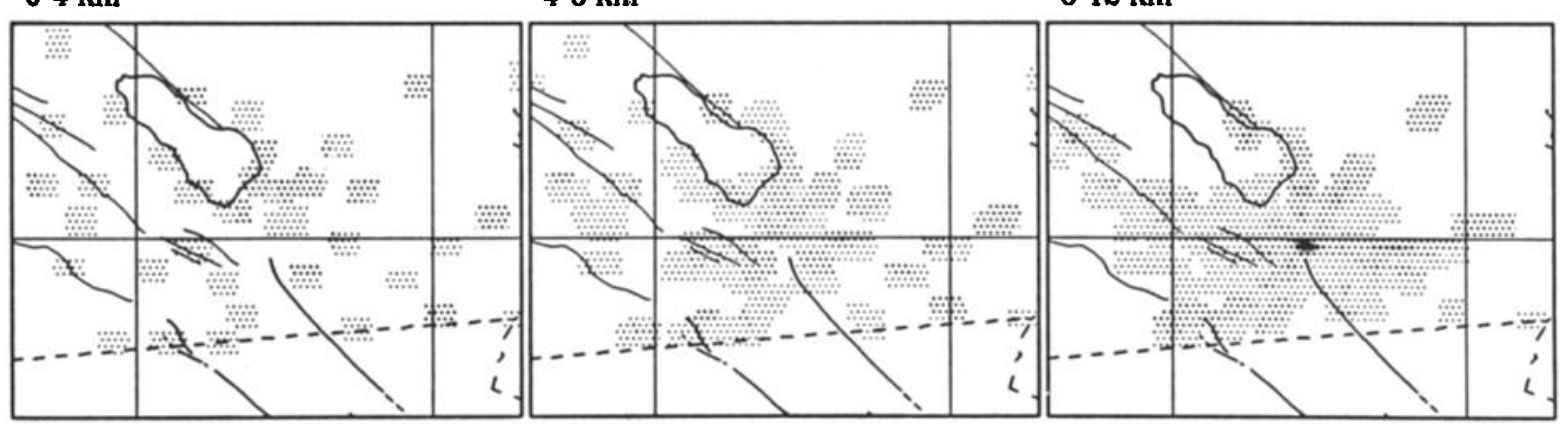

$40 \mathrm{~km}$

Imperial Valley: Resolution test on ds

\section{velocity variations}

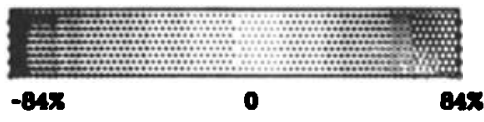

c). $0-4 \mathrm{Km}$

4-8 Km

8-12 Km

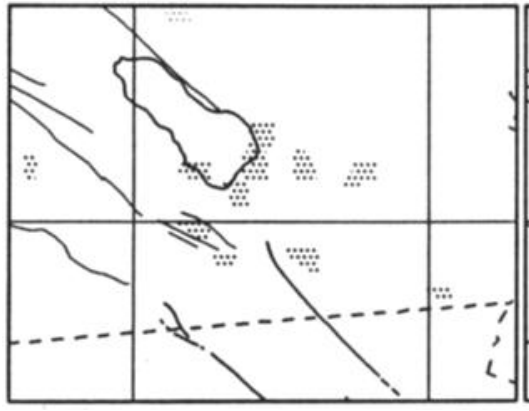

$4-8 \times m$

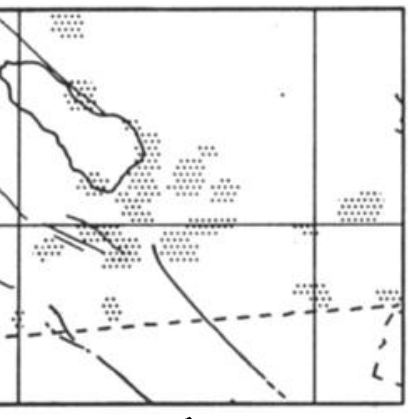

$40 \mathrm{~km}$

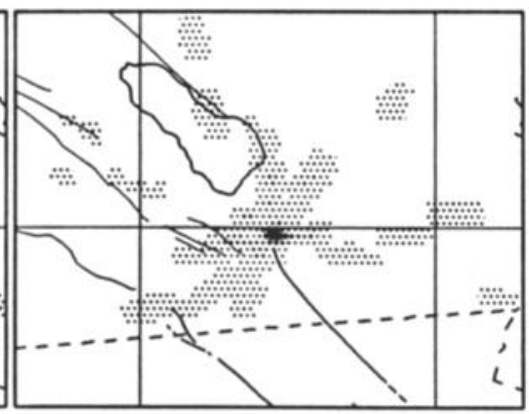

o

,

Imperial Valley: Resolution test on 1/Q

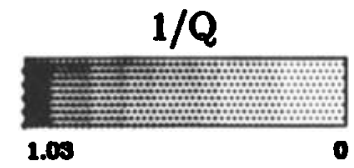

Fig. 14. (o) Location of a synthetic anomaly in the Imperial Valley study. In the travel time inversion a value of do $=1.0$ was used, and a value of $Q_{\beta}=1.0$ was used in the attenuation inversion. The block was at a depth of 8.6 $\mathrm{km}$. (b). Results of resolution test on travel time inversion for Imperial Valley: Little ray steaking is seen and over 82\% of the travel time anomaly was recovered after $\mathbf{3 0}$ iterations. e). Results of resolution test on attenuation inversion for Imperial Valley: The result is very similar to the travel time test and over $96 \%$ of the anomaly was recovered after 30 iterations.

to measure $S$ wave amplitudes from vertical component records. The $S$ waves observed on vertical component seismograms are mostly $S V$ waves which are more difficult to interpret than $S H$ waves. Furthermore, the incidence angle at the station is usually small, especially on the thick low-velocity sedimentary layers of the Imperial Valley, so that the vertical component is not very sensitive to $S$ waves. Thus the experimental setup is not ideal for our 
0-12 Km

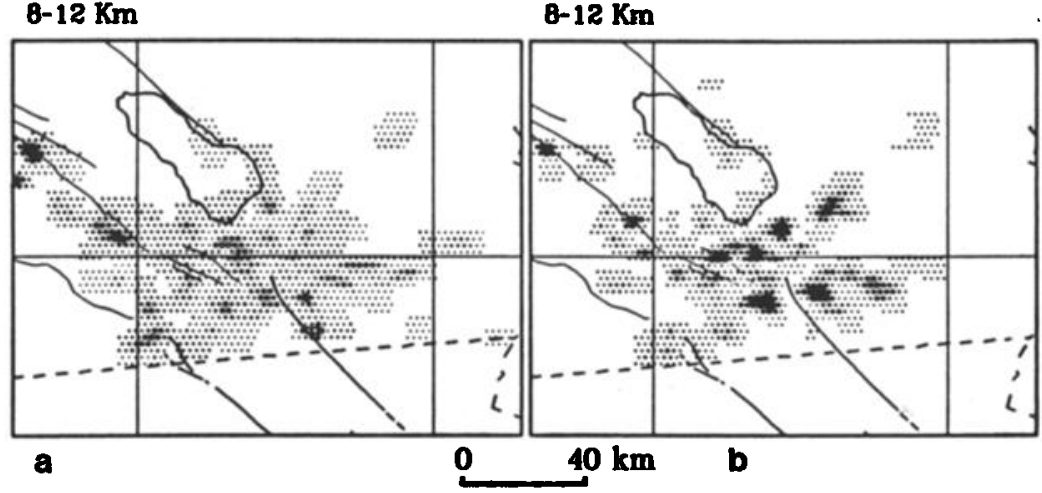

Imperial Valley: Random Noise Teet

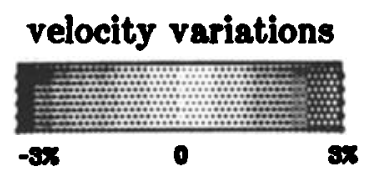

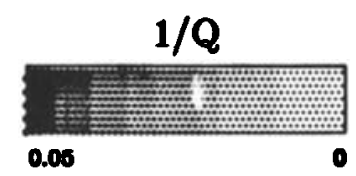

3-5 Km

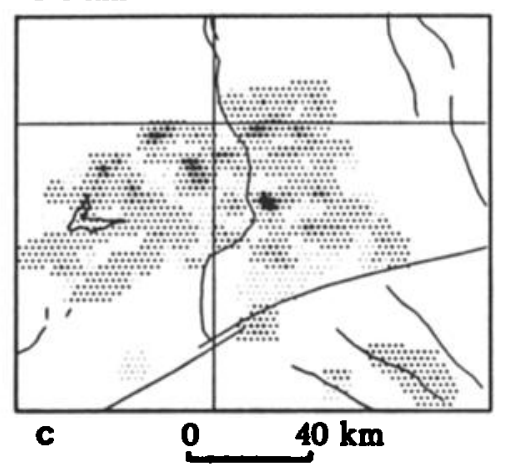

Coso region: Random Noise Test

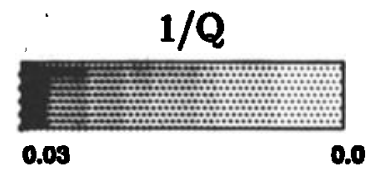

Fig. 15. Resuits of tests on inversion with random noise as synthetic data. (a) Depth slice \&-12 km of the Imperial Valley travel time test: near the vicinity of the Brawley seismic zone, no anomaly is resolved by inverting random noise as sy nthetic travel time data. (b) Depth slice $8-12 \mathrm{~km}$ of the Imperial Valley attenuation test: By inverting random noise as synthetic amplitude data, no systematic anomaly is resolved in the Brawley seismic zone. (c) Depth slice 3-5 km of the Coso test: no systematic anomaly at the location of the Indian Wells Valley anomaly resolved by inverting dats is created by the random noise input.

purpose. Admittedly, we still do not thoroughly understand the nature of these $S$ waves recorded on the vertical components. One philosophy is not to use them until we can understand them well. Perhaps this has been the prevailing philosophy among seismologists, and, for this very reason, a large amount of data has accumulated unused. We took a somewhat positive philosophy and tried to use these seismograms for mapping attenuating bodies in the crust. This could be a dangerous experiment unless we care fully examine the data. Our intuitive visual inspection of the data in the manner presented by Sanders et al. [1988] indicated that the basic patterns of $S$ wave amplitude variations are large and systematic, suggesting that they are primarily caused by localized low- $Q$ bodies in the crust.

If horizontal instruments are installed in these areas in the future, our method can be used more effectively to determine the crustal $Q$ structures. In the present study we tested the effect of the sediments on the final result by choosing different values for the parameter $k_{1} k_{2}$. As we showed carlier, the overall result remained unchanged for a large range of values of $k_{1} k_{2}$, though the details of the geometry of the anomalous bodies and the $Q$ values changed.

There is one station in the Imperial Valley area that has one horizontal component (SNRE). We examined seismograms of the events recorded at this station. The station SNR is situsted on thick sedimentary layers, and any effect of sediments on the measurement of $S V$ amplitudes should be apparent on the set of vertical and horizontal records at SNR. Since SNR is located south of the Brawley anomaly, we should have a very good constraint on the southern extent of the Brawley anomaly if $S$ waves are observed at SNR. We found that the $S$ to $P$ amplitude ratios on the vertical component are normal to partially normal on all records of the events used in the study. This suggests that the effect of sediments on the measurement is not as large as 'we initially estimated; therefore $k_{1} k_{2} \approx 1$ is probably a.good assumption, and the southern extent of the Brawley anomaly is well constrained by the existence of $S$ arrivals at SNR.

In the formulation of the attenuation tomography given by equations (4)-(12), we did not include the effect of focusing and defocusing (scattering) of the energy caused by the heterogeneity of the medium. In the actual medium, however, the wave amplitude can be amplified by focusing. As a result of this, negative values of $Q$ often resulted from the inversion. In the results shown in Figures 6, 8, 9, and 12, we have imposed a positivity constraint on the $Q$ values. Whenever negative $Q$ values were obtained, they were replaced by $Q=\infty$. In order to see the effect of this constraint, we inverted the data without the positivity constraint. As shown by Figures $16 a\left(k_{1} k_{2}=1\right)$ and $16 b\left(k_{1} k_{2}\right.$ $=1)$, the results are essentially the same as those obtained with the positivity constraint (Figures $8 a$ and 12b).

We computed the variance of the residual of the right hand side of the equation (7a) at each iteration. Between the initial and the last iterations, we obtained a variance reduction of $53 \%$ in the Coso case and $72 \%$ in the Imperial Valley, with $k_{1} k_{2}=1.0$.

The effect of focusing and defocusing is hard to assess unless we have a fully thiree-dimensional velocity model for both $P$ and $S$ waves. Such a model is also necessary for assessing the validity of our assumption that $P$ and $S$ wave geometrical spreading factors are the same. We can obtain a $\boldsymbol{P}$ wave velocity model by inverting the $\boldsymbol{P}$ travel time residuals, but with frequently missing $S$ waves, it is unlikely that we can invert the $S$ wave data, in the same fashion, to obtain $S$ wave velocity models. 
0-1 Km

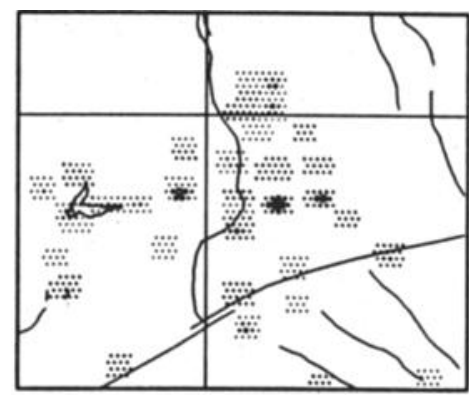

5-7 Km

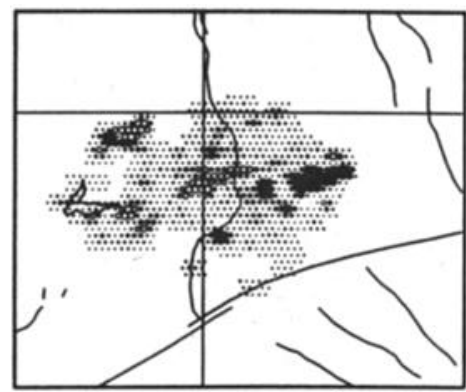

$1-3 \mathrm{Km}$

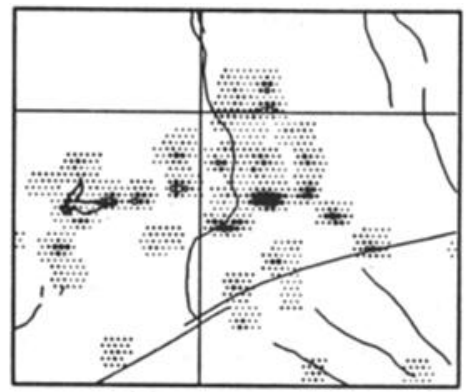

$7-9 \mathrm{Km}$

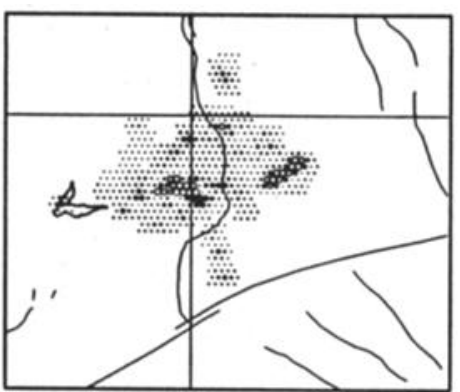

$3-5 \mathrm{~km}$

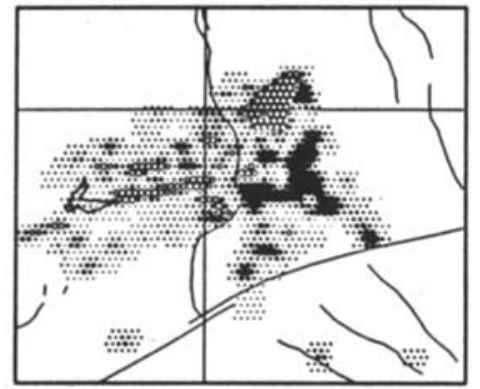

Coso region: $Q$ Invertion -re

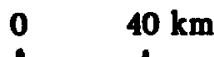

$1 / \mathrm{Q}$

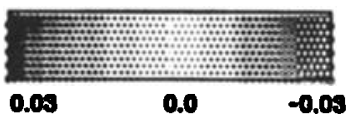

Fig. 16a. Results of attenuation inversion using $k_{1} k_{2}=1.0$ for Coso-Indian Wells region. There is no positivity constraint for $Q_{i}$ in this inversion. Notice that the geometry of anomalies resolved is the same as in the corresponding results in Figure 8 a.

There are some questions about the Brawley anomaly being in the same location as some of the events used in the study. We have shown previously that the effects of noise in the data do not result in spurious anomaly in the Brawley area. In order to show that the anomaly is not due to clustering of events in the same location, we inverted the data from only 10 out of the original 15 events. Only one event (event 1) is located in the Brawley region. Results of this inversion is shown in Figure 17b. The Brawley anomaly is still clearly imaged at the same location as that imaged by using 15 events (Figure 17a). By using 15 events we obtained a smooth pattern of anomalous areas and some anomalies seen in Figure 176 disappeared in Figure 17a. This set of results supports our conclusion that

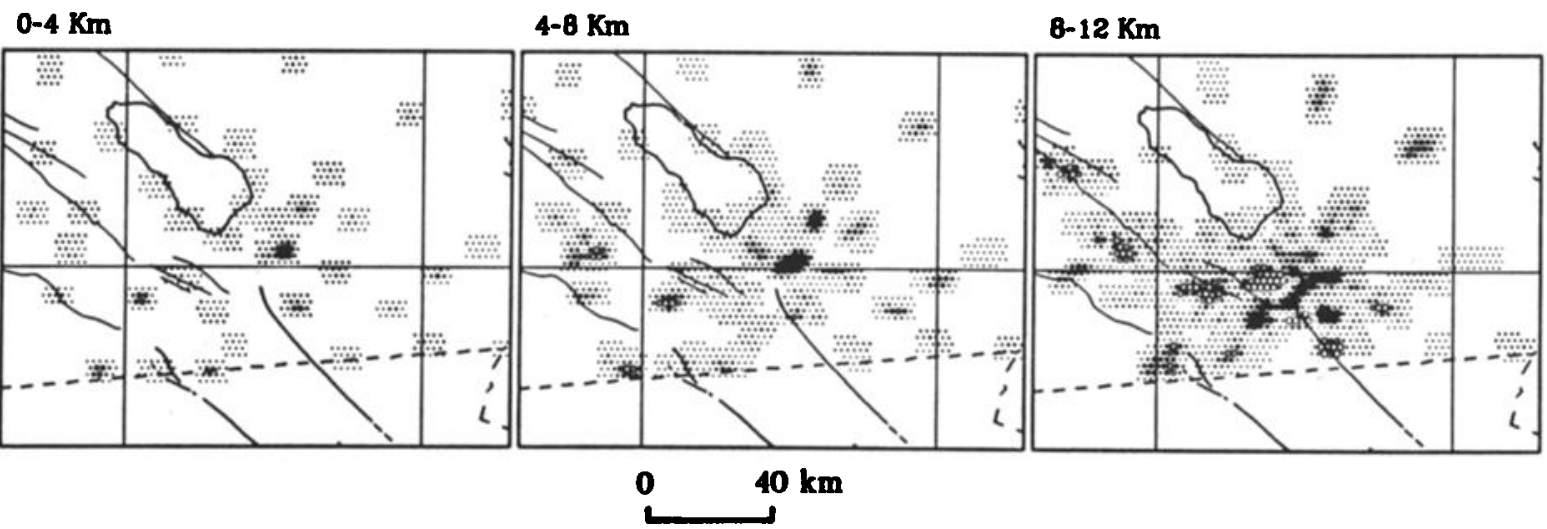

Imperial Valley: Attenuation Inversion -ve Q

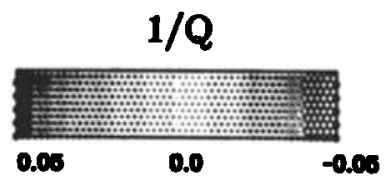

Fig. 16b. Result of attenuation inversion using $k_{1} k_{2}=1.0$ for Imperial Valley. No positivity constraint was imposed in the inversion. The geometry of the Brawley anomaly is the same as in the results in Figure $12 a$. 
a). 0-4 Km

4-8 Km 8-12 Km

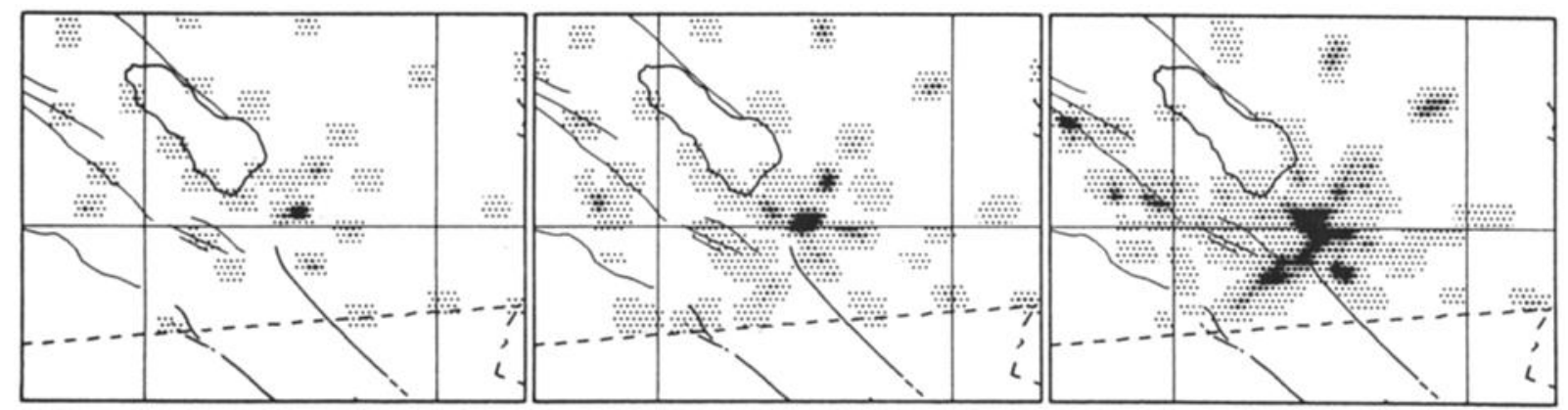

b). $0-4 \mathrm{Km}$

4-8 Km 8-12 Km

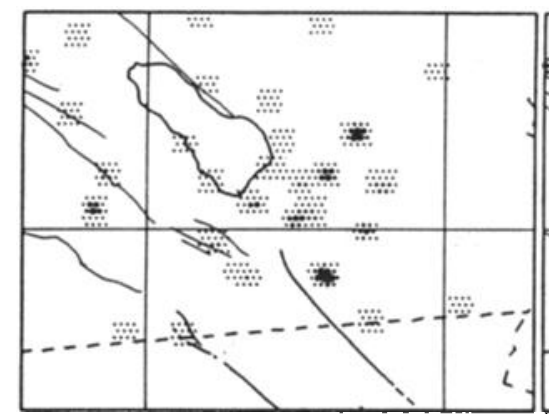

\section{8-12 Km}
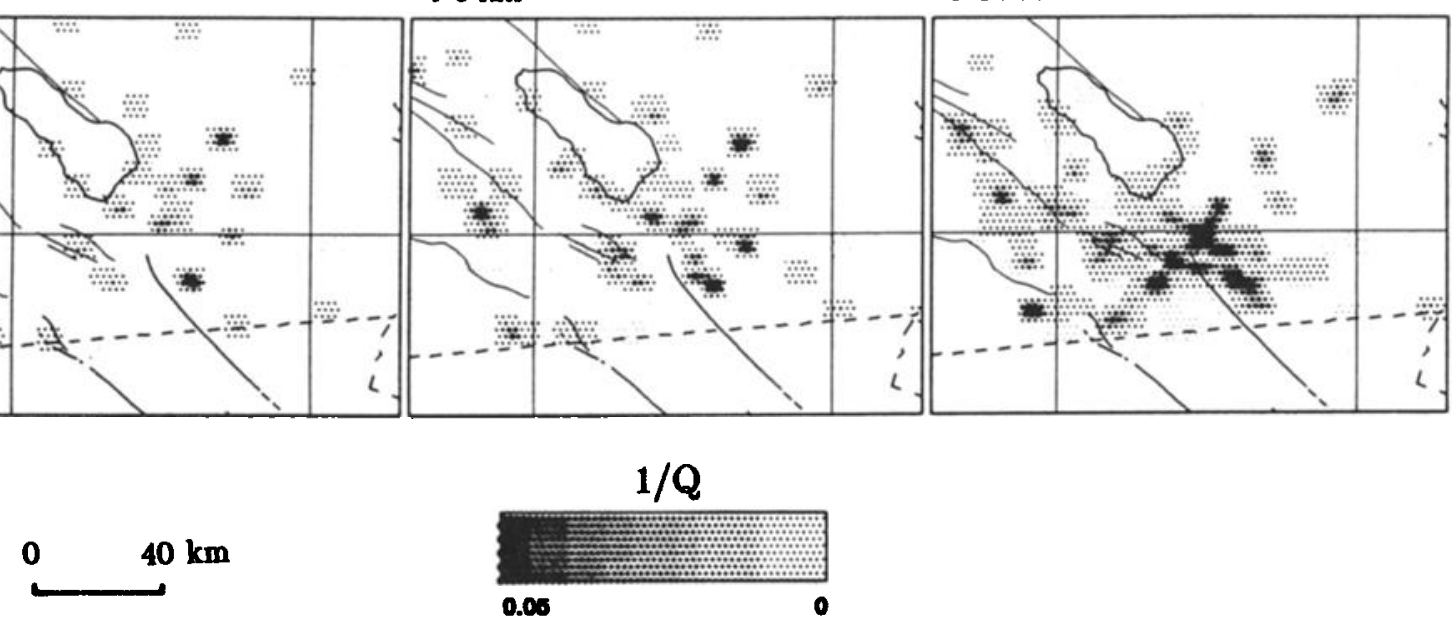

Imperial Valley: Attenuation Inversion

Fig. 17. Results of attenuation inversion with $k_{1} k_{2}=1$ by using (a) 15 events and (b) 10 events in the Imperial Valley. The 10 events used are events $1,2,5,6,7,8,9,10,11$, and 12. The general geometry of the Brawley anomaly is very similar to that imaged by using 15 events. Some anomalies shown in Figure $17 b$ are probably a result of the poorer ray coverage with less events and are spurious.

the Brawley anomaly is a real anomaly and not an artifact of inversion of the data set with many events in the area of the anomaly.

\section{CONCLUSION}

For the Indian Wells Valley, despite the use of a limited data set of only 16 earthquakes as compared to more than 400 earthquakes in the Walck and Clayton study of this region, we could resolve an attenuating body with $Q_{\beta}$ of approximately 30 in the same location as the slow velocity anomaly. This shallow attenuating body between 3 and 5 km beneath the Indian Wells Valley (IWV) extends northward close to the Coso Range. It extends down to $5-7 \mathrm{~km}$ range and to the west (Figure 8a) of Indian Wells Valley, but the anomaly north of the IWV anomaly disappears at this depth range. At the depth range of 7-9 $\mathrm{km}$, the IWV anomaly extends to the west of the Sierra Nevada fault (Figure 8a). An attenuating body east of the Indian Wells Valley could be an artifact of ray streaking in a general N$\mathbf{S}$ direction due to the poor azimuthal coverage in this vicinity (see Figure $8 c$ ).

For the Imperial Valley, 15 earthquakes were analyzed, but we resolved a slow anomaly and a highly attenuating anomaly in the region of the Brawley seismic zone. Based on the hit count map (Figure 11), we expect to have good resolution near the Brawley seismic zone, especially in the 8-12 $\mathrm{km}$ depth range. Travel time inversion images a slow velocity anomaly (about $10 \%$ slower) at a depth range of 8-12 km in the Brawley seismic zone (Figure 10). This slow anomaly extends to the $4-8 \mathrm{~km}$ depth range in the same vicinity, but the anomaly decreases in magnitude to about $5 \%$ at this depth range. Inversion of the amplitude data shows a highly attenuating body extending from $4 \mathrm{~km}$ down to $12 \mathrm{~km}$, which is also in the Brawley seismic zone. The depth range of $8-12 \mathrm{~km}$ (Figure 11a) shows a more extensive attenuating anomaly which is confined in the general area of the Brawley seismic zone. $A Q_{\beta}$ value of approximately 20 is obtained for the major attenuating body located at a depth of 8-12 $\mathrm{km}$ in the Imperial Valley. The other anomalies shown at the edge of the dense hit count area are probably artifacts of poor azimuthal coverage. According to the resolution analysis, the anomaly in the depth range of $8-12 \mathrm{~km}$ in the vicinity of Brawley is well resolved.

The shallow attenuating anomaly in Coso is probably a magmatic body, as was discussed extensively by Sanders et 
al. [1988] and is supported by the conclusions of Walck and Clayton [1987] and Walck [1988]. It is possible that the Imperial Valley attenuating body is of the same nature as the one in Coso because the values for both velocity variations and $Q_{\beta}$ are similar. However, the difference in the depth suggests some differences in the cause of such anomalies. One possibility for such a deep crustal anomaly is that it is related to the transition from upper to lower crust at a depth of about $10 \mathrm{~km}$ [Hearn and Clayton, 1986a]. This transition depth is also supported by the maximum depth of seismicity data in the area [Doser and Kanamori, 1986]. Another possibility is that it implies an active shear region. By shear region, we mean a region of shattered rocks that is undergoing large-scale shearing. Because of the presence of highly fractured rocks, seismic waves traversing the region are both attenuated and slowed down. Either magmatic or hydrothermal activity may be responsible for the anomalous structure, though the depth range of $8-12 \mathrm{~km}$ is probably less favorable for the presence of hydrothermal activity.

Acknowledgments. We thank Chris Sanders for valuable suggestions in the early stage of this study and providing us with the Coso data set and Marianne Walck for providing us with the results obtained in the Coso-Indian Wells study (Figure 7). We also thank Christof Stork for supplying the three-dimensional raytracing code. Discussions with Art Frankel, Carl Johnson, Kerry Sieh, Richard Stead, and John Vidale have been very helpful. We also thank Luciana Astiz, E. Kissling, Harold Magistrale, William Menke, Richard Stead, John Vidale, and two anonymous reviewers for reviewing the manuscript. This work is supported by USGS grant 14-08-0001-G1171 and in part by a grant from the Keck Foundation. Contribution no. 4437, from the Division of Geological and Planetary Sciences, California Institute of Technology.

\section{REFERENCES}

Anderson, D. L., and C. B. Archambeau, The anelasticity of the Earth, J. Geophys. Res., 69, 2071-2084, 1964.

Anderson, D. L., The anelasticity of the mantle, Geophys. J. R. Astron. Soc., 14, 135-164, 1967.

Doser, D. I., and H. Kanamori, Depth of seismicity in the Imperial Valley region (1977-1983) and its relationship to heat flow, crustal structure, and the October 15, 1979, earthquake, J. Geophys. Res., 91, 675-688, 1986.

Fawcett, J. A., and R. W. Clayton, Tomographic reconstruction of velocity anomalies, Bull. Seismol. Soc. Am., 74, 2201-2219, 1984.

Frankel, A., The effects of attenuation and site response on the spectra of microearthquakes in the northeastern Caribbean, Bull. Seismol. Soc. Am., 72, 1379-1402, 1982.

Fuis, G. S., W. D. Mooney, J. H. Healy, G. A. McMechan, and W. J. Lutter, Crustal structure of the Imperial Valley region, The Imperial Valley, California Earthquake of Oct. 15, 1979, U.S. Geol. Surv. Prof. Pap., 1254, 25-49, 1982

Hearn, T. M., and R. W. Clayton, Lateral velocity variations in southern California, 1, Results for the upper crust from Pg-waves, Bull. Seismol. Soc. Am., 76, 511-520, $1986 a$.

Hearn, T. M., and R. W. Clayton, Lateral velocity variations in southern California, 2, Results for the lower crust from $P n$-waves, Bull. Seismol. Soc. Am., 76, 495-509, $1986 b$.

Hill, D. P., A model for earthquake swarms, J. Geophys. Res., 82, 1347-1352, 1977.

Humphreys, E. D., R. W. Clayton, and B. H. Hager, A tomographic image of mantle structure beneath southern California, Geophys. Res. Lett., 11, 625-627, 1984.

Kobayashi, A., I. Furuya, and K. Uhira, Re-examination of magma reservoirs by spectral analysis of $P$ and $S$ waves, Geophys. Mag., 41, 173-187, 1986.

Lee, W. H. K., and J. C. Lahr, HYPO71(revised): A computer program for determining hypocenter, magnitude and first-motion patterns of local earthquakes, U.S. Geol. Surv. Open File Rep., 75-311, 1975.

Liu, H. L., Interpretation of near-source ground motion and implications, $\mathrm{Ph} . \mathrm{D}$. thesis, 184 pp., Calif. Inst. of Technol., Pasadena, 1983.

Rautian, T. G., V. I. Khalturin, V. G. Martynov, and P. Molnar, Preliminary analysis of the spectral content of $P$ and $S$ waves from local earthquakes in the Garm, Tadjikistan region, Bull. Seismol. Soc. Am., 68, 949-971, 1978.

Sanders, C. O., Location and configuration of magma bodies beneath Long Valley, California determined from anomalous earthquake signals, J. Geophys. Res., 89, 8287-8392, 1984.

Sanders, C. O., P. Ho-Liu, D. Rinn, and H. Kanamori, Anomalous shear wave attenuation in the shallow crust beneath the Coso volcanic region, California, J. Geophys. Res., 99, 3321-3338, 1988.

Sudo, Y., Attenuation of seismic wave in the Aso Caldera, Kyushu, Japan, paper presented at Hawaii Symposium on How Volcanoes Work, AGU, Hilo, Hawaii, Jan. 19-25, 1987.

Walck, M. C., Three-dimensional variations in shear struc-

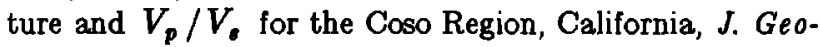
phys. Res., 98, 2047-2052, 1988.

Walck, M. C., and R. W. Clayton, $P$ wave velocity variations in the Coso region, California, derived from local earthquake travel times, J. Geophys. Res., 92, 393-406, 1987.

Walter, A. W., and C. S. Weaver, Seismicity of the Coso Range, California, J. Geophys. Res., 85, 2441-2458, 1980

Ward, R. W., and C. Y. Young, Mapping seismic attenuation within geothermal systems using teleseisms with application to the Geysers-Clear Lake region, J. Geophys. Res., 85, 5227-5236, 1980

Weaver, C. S., and D. P Hill, Earthquake swarms and local crustal spreading along major strike-slip faults in California, Pure Appl. Geophys., 117, 51-64, 1978

Wong, J., P. Hurley, and G. F West, Crosshole seismology and seismic imaging in crystalline rocks, Geophys. Res. Let., 10, 686-689, 1983.

R. W. Clayton, P. Ho-Liu, and H. Kanamori, Seismological Laboratory, 252-21, California Institute of Technology, Pasadena, CA 91125.

(Received March 18, 1987; revised December 10, 1987; accepted March 6, 1988.) 